\title{
Mit zweierlei Maß gemessen - Kalkulierte Ambivalenz in rechtspopulistischen Repräsentationen von Geschlechterverhältnissen
}

\author{
Martin Reisigl
}

Online publiziert: 7. April 2020

(C) Der/die Autor(en) 2020

Zusammenfassung Kalkulierte Ambivalenz ist ein rhetorisches Prinzip, das in Parteiprogrammen, in der politischen Werbung, in konsensorientierten Kommunikationstypen wie Gedenkreden, in diplomatischen Text- und Diskursarten sowie halbherzigen Entschuldigungen gerne verwendet wird. Es dient dazu, die Vereinbarkeit von gegensätzlichen politischen sowie ideologischen Interessen und Positionen zu suggerieren, mehrere Gruppen von Wähler*innen gleichzeitig zu adressieren, Tabus zu brechen, ohne die Verantwortung übernehmen zu müssen, und die Festlegung auf eine eindeutige Position zu vermeiden. Sie kann das Ergebnis der Mitwirkung unterschiedlicher Personen an der Textarbeit sein. Der Beitrag geht darauf ein, wie das Prinzip im rechten oppositionellen ebenso wie im Regierungspopulismus der FPÖ bei der Darstellung von Geschlechterverhältnissen angewandt wird, um die traditionelle Männerpartei als frauenfreundlichere Partei erscheinen zu lassen.

Schlüsselwörter Rechtspopulismus · Geschlecht · Kalkulierte Ambivalenz • Antifeminismus · FPÖ 


\title{
Applying Double Standards - Calculated Ambivalence in Right-Wing Populist Representations of Gender Relations
}

\begin{abstract}
Calculated ambivalence is a rhetorical principle that is keenly used in party programs, political advertising, consent-oriented genres such as commemorative speeches, diplomatic genres and half-hearted apologies. It serves the function to suggest the compatibility of diverging political and ideological interests and positions, to address different groups of voters simultaneously, to break taboos without taking the responsibility for it and to avoid a clear positioning. It can result from the involvement of different authors writing a text together. The article looks at the Austrian Freedom Party (FPÖ) and at how these right-wing populists employ - both as oppositional and as governing politicians - the principle of calculated ambivalence in their representations of gender relationships in order to make the traditional men's party appear more women-friendly.
\end{abstract}

Keywords Right-Wing Populism $\cdot$ Gender $\cdot$ Calculated Ambivalence $\cdot$ AntiFeminism · Austrian Freedom Party (FPÖ)

\section{Zum Einstieg: Frauendiskriminierung als Bestandteil des rechtspopulistischen Weltbildes}

Spezifische politolinguistische Untersuchungen zu rechtspopulistischen Darstellungen von Geschlechterverhältnissen gibt es bis jetzt kaum. Derartige Studien können allerdings sehr aufschlussreich sein. Dies sei im Folgenden am Beispiel der FPÖ aufgezeigt.

Pauschal betrachtet scheint der Befund klar zu sein: Wenn im rechtspopulistischen Weltbild Geschlechterverhältnisse repräsentiert werden, dann stellt sich rasch der Eindruck von Geschlechterdiskriminierung ein. Die rechtspopulistische Perspektive ist in der Regel eine sehr patriarchale. Sie geht mit ausgeprägtem Androzentrismus einher, sind rechtspopulistische Parteien doch ausgesprochene >Männerparteien (siehe hierzu z. B. Mudde 2007, S. 90-118; Spier 2010, S. 146-147), die sich z. T. stark aus Männerbünden rekrutieren. Beispielsweise sind Ende 2019 unter den 30 Abgeordneten der FPÖ nur fünf Frauen (das heißt 16,67\%) und gehören 40 Prozent der FPÖ-Abgeordneten Burschenschaften an. ${ }^{1}$ Rechtspopulistische Familienund Frauenpolitik trägt in der Regel reaktionäre und antifeministische Züge. Einen zentralen Programmpunkt von rechtspopulistischer und rechtsextremer Familienpolitik stellt die biopolitische Forderung dar, es möge mehr einheimische Kinder geben, damit es zu keiner Transformation der angestammten ethnisch-kulturellen Bevölkerungsstruktur komme (siehe hierzu z. B. das Parteiprogramm der AfD 2016, S. 80-87). Familienartige soziale Bindungen jenseits heterosexueller Beziehungen

\footnotetext{
1 Im Vergleich dazu weisen die Grünen Ende 2019 einen Frauenanteil von 57,69\%, die SPÖ einen Frauenanteil von $47,50 \%$, die NEOS einen Frauenanteil von $40 \%$ und die ÖVP einen Frauenanteil von 36,62\% auf. Siehe dazu https://www.parlament.gv.at/SERV/STAT/PERSSTAT/FRAUENANTEIL/frauenanteil_ NR.shtml, zuletzt abgerufen am 02-01-2020.
} 
werden abgelehnt. Machismus ist unter rechtspopulistischen Politikern ein weit verbreitetes Verhaltensmuster. Im Rechtspopulismus wird zudem häufig ein sexistischer bzw. genderistischer und heteronormativer Biologismus vertreten. ${ }^{2}$ Er beharrt strikt auf der Binarität der Geschlechter und negiert die Realität der Geschlechtervielfalt.

Viele Beispiele aus der Geschichte des Rechtspopulismus stützen diesen Gesamteindruck der Geschlechterdiskriminierung. Einleitend seien einige Beispiele zu drei Ländern angeführt.

In Italien prahlte der ehemalige Chef der Lega Nord wiederholt mit der angeblichen erektilen Potenz seiner Partei. Der 2004 und 2007 von Umberto Bossi metaphorisch geäußerte Satz »Ma la Lega Nord, ce l'ha duro! « anthropomorphisiert die Partei zum Manne. Andro- und phallozentrisch führt er die Erektionsfähigkeit als politische Kategorie ein, um Durchsetzungskraft zu signalisieren und Machtansprüche zu legitimieren. Silvio Berlusconi ließ in den Talkshows, die in seinen Fernsehsendern liefen, schon lange vor seiner politischen Karriere spärlich bekleidete Frauen gewissermaßen als dekorative Accessoires posieren, tanzen und assistieren. Eines dieser >Showgirls<, nämlich Mara Carfagna, war in Berlusconis Regierung zwischen 2008 und 2011 sogar Ministerin für Gleichstellungsfragen. Zahlreiche frauenfeindliche Äußerungen sind von Berlusconi überliefert, von den Callgirl-Partys in seiner Villa Arcore bei Mailand einmal ganz abgesehen. Der gegenwärtige Lega-Chef Matteo Salvini fiel ebenfalls wiederholt durch frauenfeindliche Angriffe auf. Beispielsweise verglich er Laura Boldrini, die Präsidentin der Abgeordnetenkammer, 2016 mit einer aufblasbaren Puppe, und 2018 fand er, dass manche Feministinnen besser eine Burka tragen sollten - implikatiert hatte er dabei: »angesichts ihres Aussehens «. Wie sehr Frauenfeindlichkeit unter Italiens Politikern quer durch die populistischen Lager grassiert, demonstrierte auch Beppe Grillo. Hasserfüllt attackierte er 2014 ebenfalls Laura Boldrini, deren kommunale Besteuerung von Immobilien ihm missfiel. Auf seinem Blog fragte er seine Leser*innen, was sie mit Boldrini machen würden, wenn sie mit ihr alleine im Auto wären. Zahlreiche Postings mit Vergewaltigungsphantasien folgten als Antwort.

Blicken wir in die USA, dann müssen wir konstatieren, dass die Vereinigten Staaten seit Anfang 2017 einen rechtspopulistischen Präsidenten haben, der trotz des Wissens um seine zahlreichen frauenverachtenden Äußerungen und sexuellen Übergriffe sein Amt auch drei Jahre später immer noch innehat.

Wenn wir uns dem Rechtspopulismus in Österreich zuwenden, dann stoßen wir auf zahlreiche Politiker der FPÖ, die in der Vergangenheit durch frauendiskriminierende Äußerungen aufgefallen sind, darunter Jörg Haider, Heinz-Christian Strache, Herbert Kickl, Peter Fichtenbauer, Johannes Hübner und Harald Vilimsky. ${ }^{3}$ Vier

\footnotetext{
2 Den mehrdeutigen Terminus Genderismus verwende ich hier in Analogie zu Sexismus, um jene Form der Diskriminierung zu bezeichnen, die asymmetrische soziale Geschlechterverhältnisse konstruiert, welche einem bestimmten Geschlecht oder mehreren Geschlechtern zum Nachteil gereichen. Der Terminus Sexismus denotiert, streng genommen, nur jene Geschlechterdiskriminierung, die sich auf biologisches Geschlecht bezieht (Sexus meint ja das biologische Geschlecht). Siehe zur kontroversen Diskussion über Begriffe wie Genderismus und Antigenderismus unter anderem Mayer/Goetz (2019, S. 210-213).

3 Verbale Frauenfeindlichkeit und Antifeminismus waren und sind in Österreich keine Besonderheit der FPÖ. Eva Rossmann hat in ihrem Buch »Unter Männern. Frauen im österreichischen Parlament« bereits 1995 eine Zwischenbilanz zu frauenfeindlichen und antifeministischen Äußerungen und Tätlichkeiten im
} 
Zitate mögen zum Einstieg genügen, um diese Frauendiskriminierung zu veranschaulichen.

In einem Artikel der Zeitschrift News vom 5. November 2011 zum Frauenbild von FPÖ-Politikern wird Peter Fichtenbauer mit den folgenden Worten zitiert (Beispiel 1):

Eine Frau ist eine Frau, und ein Mann ist ein Mann. Eine Gleichmacherei lehne ich ab, sagt der Anwalt Peter Fichtenbauer. Stammesgeschichtlich hat sich die Verteilung, dass der Mann auf die Jagd geht und die Frau sich um das Heim kümmert, als Erfolgsmodell der Gattung Homo sapiens bewährt. (Duffek 2011; https://www.news.at/a/frauenbild-fpoe-bzoe-ein-mann-310996).

Hier versucht der konservative FPÖ-Politiker, der ein Vertrauter des ehemaligen FPÖ-Chefs Heinz-Christian Strache war, zuerst mit einem essentialisierenden tautologischen Realitätstopos gegen die Gleichbehandlung von Frauen und Männern zu argumentieren, um dann mit einem Geschichtstopos, der sich auf die Evolution der Gattung des Homo sapiens bezieht, eine reaktionäre Verteilung der Geschlechterrollen zu begründen, wonach Männer phylogenetisch zu Jägern und Frauen zu Hüterinnen des Heimes prädestiniert seien. Die beiden Geschlechterstereotype reproduziert Fichtenbauer mit Hilfe des Kollektivsingulars, also der partikularisierenden Synekdoche vom Typ >Singular für Plural<. Wer sich dieses rhetorischen Mittels bedient (also der Mann und die Frau), steht von vornherein im Verdacht, vorurteilsbehaftete pauschale Urteile zu fällen, denen der Trugschluss der voreiligen Generalisierung inhäriert (vgl. dazu Reisigl 2016, S. 45-51).

Im gleichen Artikel wird der FPÖ-Parlamentarier Johannes Hübner zitiert, der 2017 wegen antisemitischer Anspielungen in einer Rede vor Rechtsextremen nicht länger als FPÖ-Kandidat für Nationalratswahlen tragbar war (Beispiel 2):

Der Drang nach Macht ist meistens nicht so ausgeprägt wie bei Männern. Das Streben danach, ein Alphatier zu sein, ist ein stark männlich geprägtes Phänomen. Männer wollen viel öfter führen und anschaffen. Aufgrund dieser testosterongesteuerten Eigenschaften würden Männer auch mehr Gesetze übertreten. Männern, bei denen die weibliche Komponente ihres Wesens stärker ausgebildet ist, fällt es wahrscheinlich leichter, sich gesetzestreu zu verhalten. Für die testosterongeprägten ist es schwieriger, sinniert Hübner. [...] Und die Frau? Hat die stark männliche Komponenten, kann sie, so behauptet Hübner, sogar Führungspositionen in der Wirtschaft einnehmen, oder auch in der Frauenbewegung. Die meisten Frauen jedoch seien vom Nestbauinstinkt geprägt. Sie suchten den >Löwenmann<, der dann im Nest sitzen soll. Das will der Löwenmann aber nicht, deshalb gibt es bei denen und bei den Alphatieren so viele Scheidungen. Denn Alphatiere sind wie im Tierreich oft polygam und haben den Drang, den eigenen Samen weit zu verbreiten. (Duffek 2011; https://www. news.at/a/frauenbild-fpoe-bzoe-ein-mann-310996)

österreichischen Parlament zusammengetragen, die von Männern verschiedener Parteien geäußert und begangen wurden, darunter auch von Politikern der ÖVP, der SPÖ und des Liberalen Forums (siehe z. B. Rossmann 1995, S. 93-121 und 139-203). 
Vorweg eine Anmerkung zur Art der Redewiedergabe in diesem Zeitschriftenartikel. Sie ist nicht immer eindeutig, sondern wechselt zwischen direkter und indirekter Wiedergabe, ohne dass das direkte Zitat klar durch Anführungszeichen markiert wäre. Sofern die Äußerungen Hübners wörtlich so gefallen sind, hat der FPÖ-Politiker eine Reihe biologistischer Metaphern und Frames verwendet, die sehr reaktionäre und diskriminierende Geschlechterverhältnisse stützen sollen. Mit der animalisierenden Metapher des Alphatiers versucht Hübner, männliches Macht-, Dominanzund Führungsstreben zu begründen. Die Naturalisierung setzt er mit einer abstrusen kausalen Argumentation fort. Hübner macht den variablen Spiegel des männlichen Hormons Testosteron für dreierlei verantwortlich: (a) Männer mit hohem Testosteronspiegel würden häufiger kriminell als Frauen. (b) Gesetzestreue Männer würden eher niedrigere Testosteronwerte als kriminelle Männer aufweisen (implikatiert ist, dass sie in gewisser Weise weniger männlich seien). (c) Frauen mit Führungsanspruch einschließlich Feministinnen würden einen erhöhten Testosteronspiegel besitzen (implikatiert ist, sie seien vermännlicht, also weniger fraulich). Es handelt sich hier selbstredend um eine trugschlüssige biologistische Argumentation, die ohne wissenschaftliche Belege auskommt. Hübner spinnt diese Naturalisierung weiter, indem er Frauen und Männer erneut animalisiert. Er belegt sie mit unterschiedlichen Tiermetaphern, die zusammengenommen eine Katachrese bilden, aus der sich die Unvereinbarkeit der beiden Geschlechter ableitet. Frauen rahmt Hübner als Nestbau-Tiere (wie z. B. Vögel, Insekten und Nagetiere es sind), Männer als im Rudel lebende männliche Raubkatzen (konkret Löwen), die mehrere Weibchen begatten, um zahlreiche und genetisch vielfältige Nachkommen zu zeugen. Während Frauen der angeborene natürliche Instinkt vorgebe, ein Nest für die Aufzucht ihrer Nachkommen zu bauen, würden Männer - löwengleich - ihren Samen möglichst weit verbreiten wollen, sei ihnen also schon von ihrer genetischen Anlage her ein polygames Bedürfnis eingeschrieben. Angesichts der Divergenz dieser angeblichen biologischen Vorgaben scheinen für Hübner Scheidungen bei Alphatieren vorprogrammiert zu sein. Wie schon bei Fichtenbauer, so ist auch bei Hübner der zweimalige stereotype Kollektivsingular (die Frau, der Löwenmann) ein rhetorischer Indikator für hochgradig trugschlüssige Pauschalurteile.

Ebenfalls biologisierend versucht der weit rechts stehende Generalsekretär der FPÖ, Harald Vilimsky - immer noch im gleichen News-Artikel vom 6. November 2011 - der Journalistin Tatjana Duffek und den Leser*innen weiszumachen, dass die körperliche Kraft von Männern diese eher für den harten Politik-Beruf prädestiniert erscheinen lasse als Frauen, die wiederum sensibler seien. Zudem bemüht Vilimsky einen locus a tempore, also ein zeitbezogenes Argumentationsschema, in Verbindung mit dem uralten Stereotyp der Gefallsucht von Frauen, um trugschlüssig zu begründen, weshalb weniger Frauen in die Politik gehen würden als Männer (Beispiel 3):

Freilich weist Vilimsky auf einen Nachteil des weiblichen Geschlechts im harten und zeitraubenden Politjob hin. Frauen sind mehr darauf aus, zu gefallen, sind demnach modeanfälliger, verwenden mehr Zeit für die Frisur und Kosmetik, klagt der General. Barbara Rosenkranz, die in der FPÖ schon lange 
ihren Mann steht, glaubt als zehnfache Mutter an die simple Wahrheit: Mädchen ziehen eben gerne schöne Kleider an, Buben wollen kein Prinzessinnenkostüm. (Duffek 2011; https://www.news.at/a/frauenbild-fpoe-bzoe-ein-mann$310996)^{4}$

Da Frauen laut Vilimsky so viel Zeit damit verbringen möchten, sich schön zu machen und mit der jeweiligen Mode zu gehen, um zu gefallen, würden sie für einen zeitintensiven Beruf in der Politik weniger übrig haben als Männer. Dieses trugschlüssige Vorurteil des Gefallbedürfnisses von Frauen ist nicht weit entfernt vom archaischen Stereotyp der Frauen als Verführerinnen. Das Frauenbild, das sich aus den hier wiedergegebenen Wortspenden von FPÖ-Politiker*innen speist, ist meilenweit entfernt von jedwedem modernen Frauenbild, das Emanzipation und Gleichberechtigung für selbstverständlich hält.

Eben dieses frauendiskriminierende Verführungsmotiv ist im nächsten Beispiel, das dem schon erwähnten Zeitschriftenartikel entnommen ist, multimodal zum Ausdruck gebracht und journalistisch inszeniert. Darin wird der damalige FPÖ-Obmann Heinz-Christian Strache zitiert. Er lehnt Frauenquoten als überflüssig ab, weil sich Frauen über kurz oder lang durchsetzen würden, wenn sie qualifiziert seien (Beispiel 4):

In Straches ganzem Umfeld sind nur erfolgreiche Frauen, erzählt der Blaue stolz. Ich fühle mich von starken Frauen angezogen. Nur schwache Männer suchen Hascherln. Ich mag selbstbewusste Frauen mit einem freien, kritischen Geist. Nur zu unabhängig und eigensinnig sollte die Frau nicht sein [sic!] Barbara Rosenkranz musste der FPÖ-Parteichef im Wahlkampf mehrmals zurückpfeifen. (Duffek 2011; https://www.news.at/a/frauenbild-fpoe-bzoe-ein-mann310996)

Wieder ist die Perspektive der Journalistin nicht durchgehend von der Perspektive des Politikers getrennt, der hier zitiert wird. Im vorletzten Satz dieser Textpassage, auf den interpunktionslos der letzte Satz folgt, stellt sich der Eindruck von erlebter Rede ein, bei der nicht mehr klar ist, was Strache denn tatsächlich wörtlich gesagt hat. Es ist anzunehmen, dass die einschränkende deontische Passage (»Nur zu unabhängig und eigensinnig sollte die Frau nicht sein«) eine ironische Hinzufügung darstellt, die aus der Feder der Journalistin und nicht aus dem Mund des FPÖ-Chefs stammt. Wenn wir dieses Verschwimmen der Perspektiven beiseite lassen, dann entnehmen wir dem Zitat, dass in Straches rechtspopulistischem Weltbild die Geschlechterverhältnisse so organisiert sind, (a) dass es starke Frauen (selbstbewusste Frauen mit einem freien, kritischen Geist) und schwache Frauen (Hascherln) gibt, (b) dass es starke Männer (die sich von starken Frauen im Sinne Straches angezogen fühlen) und schwache Männer gibt (die sich von schwachen Frauen, d.h. Hascherln angezogen fühlen) und (c) dass sich Strache selbst als starken Mann einstuft, der sich von starken Frauen angezogen fühlt (man beachte den Plural und man beachte, dass die Verbalmetapher bestimmte Frauen zu Magneten macht, die als physikalische

\footnotetext{
${ }^{4}$ Die drei eben wiedergegebenen Zitate werden auch in Scharsach (2017, S. 96-97) angeführt, allerdings leicht verändert.
} 
Kraft eine anziehende Wirkung auf den FPÖ-Chef ausüben). Straches Geschlechterweltbild ist zweifach zweigeteilt: in »weiblich« versus »männlich« und »stark« versus »schwach«. Strache wertet: Für Schwache, egal ob Männer oder Frauen, hat er wenig mehr als Bedauern übrig, das drückt sich im versächlichenden Diminutiv »Hascherln« in der Bedeutung von >armen, ängstlichen Frauen« aus. Aus solcher Perspektive spricht ein Sozialdarwinismus, der in Konflikt steht zum populistisch vielbeschworenen Einsatz für die kleinen Leute. Wie wenig sich Strache als Politiker für die kleinen Leute einsetzte, wurde seit der Ibiza-Affäre ab Mai 2019 (siehe dazu Obermaier/Obermayer 2019) erst so richtig deutlich. Die Affäre machte übrigens auch deutlich, dass Strache Homosexualität für etwas Abnormales und westlich Dekadentes hält (Obermaier/Obermayer 2019, S. 43).

Untermalt werden Straches Aussagen mit einem vielsagenden Bild (siehe Abb. 1). Dieses vorsätzlich gewählte Bild lässt sich als ironischer journalistischer Kommentar zur verbalen Äußerung Straches lesen. Die visuelle Kontextualisierung von Straches Äußerung in seiner Lebenswelt als Politiker, der sich gerne in Diskotheken vergnügt, dort aber auch politische Werbung betreibt, verleiht Straches Worten eine ambivalente bis konträre Bedeutung. Auf dem Bild sehen wir, wie Heinz-Christian Strache in einer Diskothek einer freizügig gekleideten blonden Frau ein Autogramm auf die obere Seite ihres Busens schreibt, den die Frau Strache entgegenstreckt und auf den die Frau selbst auch hinunterblickt, um zu sehen, was Strache schreibt. Die Frau gibt sich als Fan Straches zu erkennen. Sie hält einen Werbeflyer mit Straches Konterfei in der Hand, unter dem der folgende Zweizeiler geschrieben steht: »Sie sind gegen IHN, weil ER für EUCH ist. « Strache selbst trägt ein weißes T-Shirt, auf dem er in ambivalenter strategischer Anlehnung an den marxistischen Revolutionär Che Guevara als »HC StraCHE« inszeniert ist (siehe dazu auch Reisigl 2012b, S. 146-148). Stellt man das Bild mit dem Untertitel des Artikels in einen Zusammenhang (»Zwischen Discohäschen und Mutterkreuz. Was denken rechte Politiker über Frauen?«), dann bringen die Leser*innen des Artikels die abgebildete Frau schnell mit dem im Titel verwendeten animalisierenden Diminutiv »Discohäschen« in Verbindung. Es fragt sich nun, wie die Äußerung Straches zu der auf dem Bild dargestellten Frau passt. Das Bild deutet die Prädikation »selbstbewusst « um zu >sich verführerisch sexualisierend<, und die Prädikation »frei« verbindet sich mit dem körperlich performierten 〉Exhibitionismus $<$. Der »freie, kritische Geist«, von dem Strache spricht, lässt sich mit dem Bild der Frau, die sich selbst zum Sexualobjekt degradiert, schwerlich vereinbaren. ${ }^{5}$

Die bisherige exemplarische Zusammenschau liefert den Befund, dass rechtpopulistische Politiker*innen das Verhältnis zwischen den Geschlechtern gerne androzentrisch, biologistisch-binär, chauvinistisch, genderistisch oder sexistisch, homophob und heteronormativ perspektivieren. Die tendenziell frauendiskriminierende Haltung

\footnotetext{
5 An dem Bild ist in intertextueller Hinsicht bemerkenswert, dass der populistische Slogan »Sie sind gegen IHN, weil ER für EUCH ist.« ein Plagiat darstellt. Der Slogan wurde 1994 in Jörg Haiders Wahlkampf gebraucht. 2008 rezyklierte die FPÖ diesen Spruch, um für Strache und gegen Haider und seine Partei des BZÖ zu werben. Nicht genug damit, wurde der Slogan jüngst von der rechtspopulistischen AfD für den Thüringer Landtagswahlkampf im Oktober 2019 verwendet. Dieses Fremdplagiat ging sogar so weit, dass Björn Höcke auf dem Wahlplakat die gleiche Körperhaltung wie Jörg Haider seinerzeit vor 25 Jahren einnahm.
} 
Abb. 1 Die ironische visuelle Kommentierung von Straches angeblicher Präferenz für »selbstbewusste Frauen mit einem freien, kritischen Geist« (Duffek 2011; https://www. news.at/a/frauenbild-fpoe-bzoeein-mann-310996)

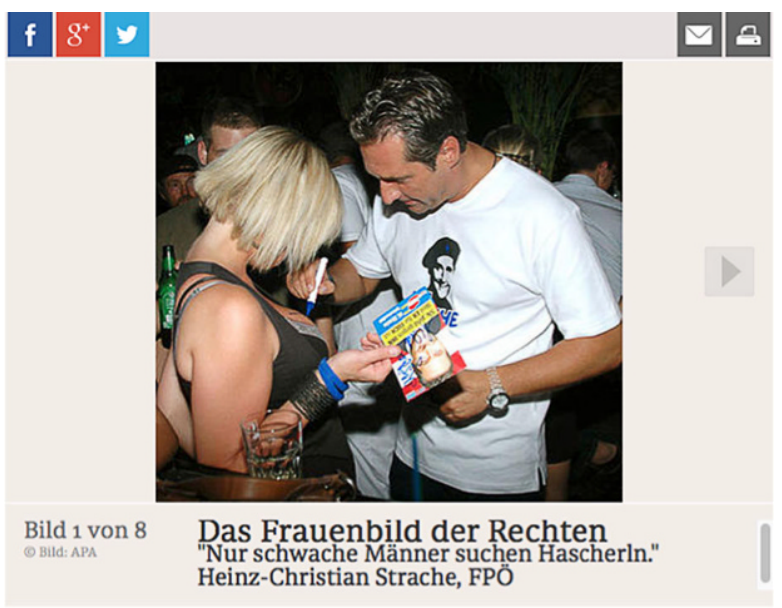

führt denn auch dazu, dass Frauen insgesamt deutlich weniger häufig rechtspopulistisch wählen als Männer (siehe hierzu in Bezug auf die frühere FPÖ schon Rösslhumer 1999, S. 30-31; bei den Nationalratswahlen 2019 wählten fast doppelt so viele Männer als Frauen die FPÖ (nämlich $21 \%$ gegenüber $11 \%$ ); siehe dazu die Wahlanalyse von SORA 2019, S. 5).

Es fragt sich an diesem Punkt allerdings, ob das schon alles ist, was über rechtspopulistische Perspektiven auf Geschlechterverhältnisse festgestellt werden kann. Die einschlägige Forschungsliteratur erweckt zuweilen den Eindruck, dass es zur Thematik nicht mehr zu sagen gebe. Jedoch wird das Bild komplexer, wenn man tiefer in die Materie eintaucht. Bei genauerem Hinsehen zeigt sich in rechtspopulistischen Darstellungen von Geschlechterverhältnissen eine kalkulierte Ambivalenz. Ziel meiner weiteren Ausführungen ist es, diese kalkulierte Ambivalenz am Beispiel der FPÖ detaillierter herauszuarbeiten. Dies wird auf der Basis einer konzisen Darlegung des von mir im Laufe der letzten zwanzig Jahre erarbeiteten Begriffs des Rechtspopulismus in Abschnitt 2 und auf der Grundlage einer Bestimmung und Erweiterung des von Josef Klein 1996 eingeführten Begriffs der kalkulierten Ambivalenz in Abschnitt 3 geschehen. An die theoretische Ausarbeitung der beiden Begriffe schließt in Abschnitt 4 die Analyse von acht konkreten Beispielen zur FPÖ an. Ein Fazit rundet meinen Beitrag dann ab.

\section{Zum Begriff des Rechtspopulismus}

Zum Begriff des Rechtspopulismus wurde in der einschlägigen sozialwissenschaftlichen Literatur viel geschrieben. Mein Verständnis hat sich in den letzten zwanzig Jahren in Auseinandersetzung mit dieser Literatur sowie dem sich wandelnden empirischen Forschungsgegenstand - insbesondere in Österreich, das auf eine mehr als 30-jährige Tradition des Rechtspopulismus zurückblicken kann - langsam ausdifferenziert. In mehreren Arbeiten ist die schrittweise Erweiterung meiner Konzeption 
von Rechtspopulismus festgehalten (Reisigl 2002, S. 149-153; Reisigl 2012a, S. 312-316; Reisigl 2012b, S. 141-145; Reisigl 2014, S. 71-78). Von Karin Priester habe ich aus Schriften (siehe z. B. Priester 2007, S. 9-45 und Priester 2012) ebenso wie in sehr anregenden interdisziplinären face to face-Diskussionen im Rahmen von drei Workshops, die Paula Diehl 2011 und 2012 in der Villa Vigoni am Comer See organisiert hat, vor allem in soziologischer Hinsicht gelernt, dass bei der theoretischen und empirischen Untersuchung von Rechtspopulismus auf alle Fälle auch die sozialen Träger*innen einbezogen werden müssen und dass eine rein formale oder stilbezogene Begriffsbestimmung zu kurz greifen würde. Aus den kontrovers diskutierten Versuchen, dem sperrigen Phänomen des Populismus mit dem Ideologiekonzept beizukommen, habe ich gelernt, dass Populismus keine Ideologie im klassischen Sinne darstellt und im Grunde auch keine dünne oder schwache Ideologie, wie etwa Mudde (2004, S. 544) annimmt. Vielmehr konstituiert sich Populismus als ideologischer Verschnitt mit variablen thematischen Schwerpunkten. Auch wenn eine sprachwissenschaftliche Auseinandersetzung mit (Rechts)Populismus die Frage nach dem Wie stets großschreibt, also die Frage nach der Art des rhetorischen Artikulationsmodus des (Rechts)Populismus, so steht doch außer Frage, dass immer auch das Was, also die inhaltliche Dimension des Populismus berücksichtigt werden muss, um neben Gemeinsamkeiten und Überschneidungen auch die Unterschiede zwischen einem Links- und Rechtspopulismus angemessen in den Blick zu bekommen. >Artikulation < (von lat. articulo in der Bedeutung von ich gliedere) verstehe ich daher auch als inhaltliche Gliederung des populistischen Weltbildes innerhalb des dreidimensionalen politischen Raums in ein Oben und Unten, ein Links und Rechts sowie ein Vorn und Hinten; und der politische Raum wird zudem durch eine Grenzziehung zwischen einem Innen und Außen konstituiert. Eine politikwissenschaftliche und politolinguistische Einordnung sollte sich außerdem dafür interessieren, aus welcher Position im politischen System sich Populismus artikuliert (ob als oppositioneller Populismus oder als Regierungspopulismus) und was bei einem etwaigen Positionswechsel innerhalb des politischen Systems passiert, wie sich also eine rechtspopulistische Partei verändert, wenn sie von der Oppositions- auf die Regierungsbank wechselt.

Zusammengefasst gehe ich von folgendem Begriffsverständnis aus: Populismus ist ein inhaltsbezogener und medienbasierter Modus der politischen Artikulation, (a) der als komplexes Syndrom auf eine Krise der politischen Repräsentation reagiert, (b) der sich auf eine vorgestellte Gemeinschaft des Volkes beruft und (c) an eine soziale Gruppe von Träger*innen gebunden ist, (d) wobei sowohl das Volkskonstrukt als auch die sozialen Träger*innen je nach ideologischer Ausrichtung variieren. Beispielsweise konstruiert der Rechtspopulismus das Volk gerne als Nation oder bedrohten Mittelstand und der Linkspopulismus als sozioökonomische Klasse der Arbeitenden. Allerdings werden aus wahltaktischen Gründen die Grenzen zwischen links und rechts immer wieder verwischt, etwa wenn sich die FPÖ als soziale Heimatpartei in Szene setzt, um auch im Lager der Sozialdemokratie zu wildern. Insofern arbeitet Populismus mit einer synkretistischen Kombination heterogener ideologischer Elemente, ohne selbst ein kohärentes ideologisches Glaubenssystem anzubieten, das ein differenziertes Modell des Status quo, ein klar spezifiziertes Modell des Status desideratus und ein ausgearbeitetes Modell der Via optima hin zum 
Status desideratus enthält. All das wäre für eine prototypische Ideologie charakteristisch.

Will man den klassischen oppositionellen Rechtspopulismus näher kennzeichnen, so lässt sich sagen, dass er zwei Antagonismen ins Spiel bringt: Zum einen beziehen sich Populist*innen affirmativ und rechtfertigend auf das Volk, das es zu vertreten gelte. Zum anderen greifen sie interne und externe Feinde und Feindinnen an. Zu den internen Feinden und Feindinnen zählen auf der vertikalen Achse prototypisch die da oben und auf der horizontalen Achse z. B. die Linken, und - um zu unserem Thema zurückzulenken - Feministinnen sowie die Schwulen-, Lesben, Bi-, Queerund Transgenderbewegungen. Die externen Feinde und Feindinnen, die von Rechtspopulist*innen an die Wand ihres schematischen Weltbildes gemalt werden, sind die Ausländer und Ausländerinnen, gegenwärtig vor allem der Islam und die EU, manchmal Juden und Jüdinnen usw.

Will man die eben angesprochene räumliche Indexikalität des oppositionellen Rechtspopulismus geometrisch visualisieren, dann lässt sich die folgende Figur entwerfen (Abb. 2). Die dritte räumliche Achse bezieht sich in der Darstellung auf die zeitlich gedeutete Vorwärts- oder Rückwärtsbewegung, also auf die in der Politik relevant gesetzte Frage der Fort- oder Rückschrittlichkeit. Diese Frage wird im Rechtspopulismus oft mit einer antimodernistischen Verklärung von Heimat und Tradition und einer reaktionären bzw. regressiven Gesellschaftsutopie beantwortet (vgl. hierzu auch Weidacher in diesem Band) - ganz nach dem Motto >Zurück in die Zukunft!<, mit dem eine Rückwärtsgewandtheit der Zukunftsorientierung ausgedrückt ist.

Vor dem Hintergrund der These, dass Rechtspopulismus als genuin vergeschlechtlichtes Phänomen aufzufassen ist (Sauer 2017, S. 4), können wir allgemein fragen, wie im politischen Raum, den der Rechtspopulismus entwirft, Geschlechterverhältnisse repräsentiert werden. Mindestens fünf Bereiche lassen sich in diesem Raum

Abb. 2 Räumliche Indexikalität des oppositionellen Rechtspopulismus

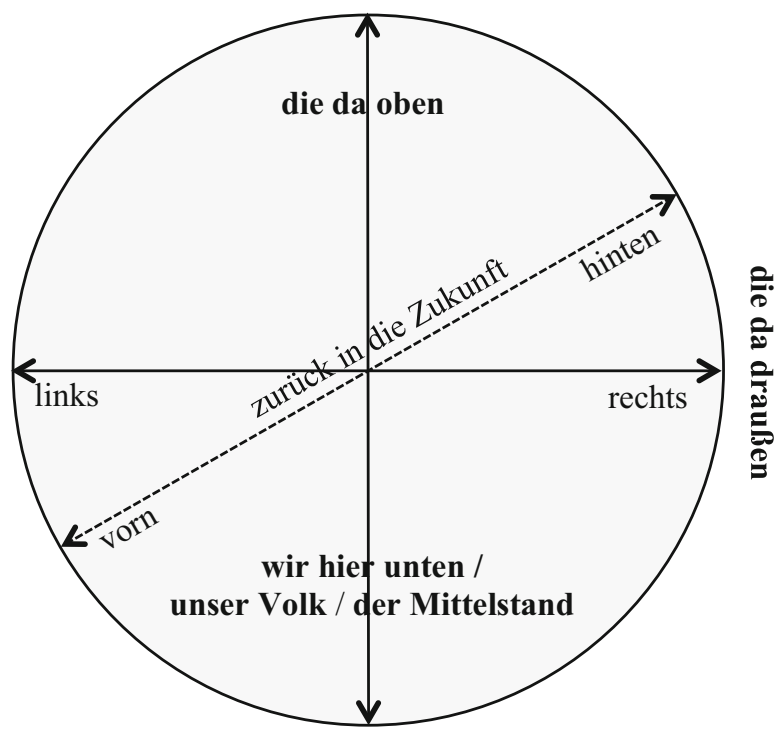


ausmachen, die sich auf die Kategorie des Geschlechts beziehen. Erstens wird das Volk immer wieder synekdochisch als >der kleine Mann (von der Straße)< imaginiert, also ein androzentrisches Freundbild konstruiert. Die >kleine Frau < findet sich im populistischen Weltbild dagegen kaum explizit repräsentiert. Zweitens zeichnet der Rechtspopulismus prototypisch rückwärtsgewandte biologistische und heteronormative Frauen- und Männerbilder. Darauf aufbauend erscheint $>$ das Volk $<$ dann als ethnopluralistisches, heterosexuell generiertes, natürliches und in sich homogenes Konstrukt (siehe dazu auch Sauer 2017, S. 12). Andererseits finden sich drittens allgemeine Bekenntnisse zur Gleichberechtigung von Frauen und Männern. Viertens attackiert der Rechtspopulismus immer wieder Feminismus und die LGBTBewegungen als interne Feindinnen und Feinde (manchmal auch metonymisch pathologisierend mit dem Begriff des Genderwahns) und artikuliert er reaktionäre Homophobie. Fünftens betreibt er aus fremdenfeindlichen Gründen die »Ethnisierung des Sexismus « (siehe zu diesem Begriff M. Jäger 1996, S. 155-293) und kritisiert die Unterdrückung von Frauen durch patriarchale Migranten. Wir sehen also, dass der Rechtspopulismus zwar insgesamt eine klare geschlechterdiskriminierende Schlagseite aufweist, es finden sich aber auch strategische Äußerungen, die sich gegen die Diskriminierung von Frauen richten. Damit stellt sich eine Ambivalenz ein, die es näher zu betrachten gilt.

\section{Zum Begriff der kalkulierten Ambivalenz}

In der Politik wird oft strategisch mehrdeutig formuliert. Wer dort das Prinzip der kalkulierten Ambivalenz anwendet, nimmt Widersprüche in Kauf, möchte diese aber möglichst verschweigen. Politolinguistik, die an Widersprüchen in der politischen Kommunikation interessiert ist, wird die kalkulierten Ambivalenzen in den Blick nehmen. Sie wird herauszuarbeiten versuchen, inwiefern kalkulierte Ambivalenz, die in politischen Diskursen geäußert wird, zu konfligierenden Geltungsansprüchen der Wahrheit und normativen Richtigkeit führt. Auch wird sie nach den Gründen für und Funktionen von kalkulierter Ambivalenz forschen.

Der Terminus der kalkulierten Ambivalenz, wurde von Josef Klein 1996 in einem äußerst kenntnis- und aufschlussreichen Text über Parteiprogramme eingeführt (Klein 1996). Im Anschluss an Klein habe ich versucht, das Konzept auch auf andere Bereiche der politischen Kommunikation zu beziehen und als eines von zehn Prinzipien der rechtspopulistischen Rhetorik zu bestimmen (Reisigl 2002, S. 168-174). Sehen wir uns an, wie Klein das Prinzip der kalkulierten Ambivalenz am empirischen Gegenstand von Parteiprogrammen begrifflich fasst, bevor das Konzept eine Ausweitung erfährt.

Strategisch ambivalentes Formulieren erfüllt nach Klein primär die Funktion, unvereinbare Positionen als vereinbar darzustellen:

Die simpelste Technik ist die Bejahung jeder der beiden Positionen, ohne ihre Unvereinbarkeit zu thematisieren. So heißt es im Berliner Grundsatzprogramm der SPD (1989): Wir bejahen die Bundeswehr und die Wehrpflicht und drei Sätze weiter: Wir achten das Engagement von Pazifisten [...] Sie haben einen 
legitimen Platz in der SPD (SPD 1990, 13). Was fachunkundige Leser nicht bemerken, oder wenn sie es bemerken, als Widersprüchlichkeit kritisieren dürften, versteht der fachkundige Leser als Konsequenz aus einer [...] Regel (Klein 1996, S. 206).

Klein spezifiziert insgesamt drei Regeln der kalkulierten Ambivalenz, die in Parteiprogrammen befolgt werden. Die erste Regel gibt vor, dass bei unverträglichen und kompromissresistenten Diskurspositionen zweier Gruppen, die beide von einer politischen Partei gleichermaßen angesprochen, umworben oder gewonnen werden sollen, die werbende Partei am besten versuchen möge, zu beiden Positionen positiv Stellung zu beziehen, ohne auf die Inkompatibilität der Positionen hinzuweisen (Klein 1996, S. 206). Die zweite Regel besagt, dass eine Partei, die sich programmatisch neu ausrichtet, um neue Wähler*innen anzusprechen, zusätzlich zur Betonung der Neuorientierung immer auch Kontinuität und Traditionsbewusstsein signalisieren sollte, um bisherige Gruppen von Wählerinnen und Wählern nicht zu verlieren. Diese Regel dient dazu, im politischen Prozess der Mehrfachadressierung Veränderung und Kontinuität gleichermaßen zu vermitteln (Klein 1996, S. 207). Die dritte Regel schließlich, die Klein formuliert, legt fest, wie die Gewichtung von Kontinuität und Veränderung in Parteiprogrammen sprachlich ausgedrückt werden kann. Sie besagt, dass die konkrete Benennung politischer Zentralfragen in dieser Textart mehr Gewicht besitzt als die abstrakte Thematisierung allgemeiner symbolischer Werte und Ziele. In diesem Sinne geht inhaltliche Konkretion und Spezifik in Parteiprogrammen vor Abstraktion (Klein 1996, S. 207). Wer diese Regeln der kalkulierten Ambivalenz kennt, wird Parteiprogramme differenzierter betrachten als die, die von ihnen keine Kenntnis haben. Wer sich außerdem vergegenwärtigt, dass die Genese von Parteiprogrammen dadurch geprägt ist, dass in der Regel mehrere Autor*innen aus unterschiedlichen parteiinternen Positionen, die durchaus auch konträr sein können, an der Konzeption und Ausformulierung des konkreten Textes mitwirken, kann die partielle inhaltliche Widersprüchlichkeit und Inkohärenz, die sich in Parteiprogrammen findet, leichter nachvollziehen und als etwas verstehen, was von den Autor*innen bewusst in Kauf genommen wird.

Allerdings sind kalkulierte Ambivalenzen nicht nur für Parteiprogramme prägend. Es lohnt sich, kalkulierte Ambivalenzen auch in weiteren Bereichen der politischen Kommunikation aufzuspüren. Sie tauchen in der politischen Kommunikation nämlich in verschiedensten Kommunikationstypen der politischen Werbung auf und sind zudem in konsensstiftenden Genres wie z.B. Gedenkreden anzutreffen, in denen gleichzeitig politische Kontinuität und Diskontinuität zum Ausdruck gebracht werden soll. Dass sie auch in diplomatischen Texten vorkommen, in denen gegensätzliche politische Interessen unter einen Hut gebracht werden müssen und eine eindeutige Entscheidungsfindung nicht möglich ist, liegt nahe. Wo in Texten oder Diskursfragmenten halbherzige Zugeständnisse an Opponent*innen gemacht werden, wird ebenfalls gerne strategisch ambivalent formuliert.

Von besonderer Brisanz ist die Anwendung des Prinzips der kalkulierten Ambivalenz in der rechtspopulistischen Rhetorik. Diese Brisanz hat viel zu tun mit einem oft problematischen Verhältnis zur Vergangenheit (also mit einer bedenklichen historischen Kontinuität), aber etwa auch mit Tabubrüchen, für die ein*e Politiker*in 
nicht zur Verantwortung gezogen werden will, durch die aber gewollt ein Skandal ausgelöst wird, mit dem Medienaufmerksamkeit generiert werden kann. Kalkulierte Ambivalenz ist oft auch auf einen opportunistischen Eklektizismus zurückzuführen, der zu ideologischem Synkretismus führt. Grundsätzlich verdankt sich kalkulierte Ambivalenz häufig einem Buhlen um Wähler*innen mit unterschiedlichen Interessen, stellt sie also ein Manöver persuasiver Mehrfachadressierung dar.

Kalkulierte Ambivalenz folgt einer Verknüpfungslogik des Sowohl-als-auch. Diese konjunktive Logik sieht der Soziologe Ulrich Beck in seinem Buch »Die Erfindung des Politischen« (1993, S. 9-23) als charakteristisch für das 20. Jahrhundert an, während das 19. Jahrhundert Beck zufolge noch durch rigide Disjunktivität gekennzeichnet gewesen sei:

Während das 19. Jahrhundert vom entweder-oder regiert wurde, sollte das 20. Jahrhundert der Arbeit am und gelten. Dort Trennung, Spezialisierung, das Bemühen um Eindeutigkeit, Berechenbarkeit in der Welt - hier: Nebeneinander, Vielheit, Ungewißheit, die Frage nach dem Zusammenhang, Zusammenhalt, das Experiment des Austausches, des eingeschlossenen Dritten, Synthese, Ambivalenz. (Beck 1993, S. 9)

Ambivalenz führt - auch in der Politik - zu Komplexität, Unklarheit und Unsicherheit. Dem steht das Bedürfnis nach Eindeutigkeit und Einfachheit gegenüber. Rechtspopulistische Parteien reagieren auf dieses Bedürfnis von verunsicherten Wähler*innen, die nach Orientierung suchen, indem sie die Komplexität der Wirklichkeit drastisch reduzieren und vermeintlich einfache und eindeutige Lösungen anbieten (Reisigl 2002, S. 166). Wollen sie im Kampf um »Gefolgschaft« allerdings an Einfluss gewinnen, können sie die radikale Eindeutigkeit nicht durchhalten, da sie mit ihr nur ein kleines Segment von Wähler*innen erreichen. Daher greifen sie zum rhetorischen Prinzip der kalkulierten Ambivalenz. Der politische Erfolg einer Partei wie der FPÖ beruht wesentlich auf diesem Prinzip.

Eben diese Strategie des Doublespeak ist keineswegs neu in der politischen Kommunikation. Sie wurde z. B. bereits von der nationalsozialistischen Bewegung angewandt, um Mehrfachadressierungen vorzunehmen. Das zeigt sich schon am Parteinamen, der vermeintlich Sozialistisches mit Deutsch-Nationalistischem verbindet (Reisigl 2002, S. 143 f., siehe dazu auch Eitz/Engelhardt (2015, S. 308-313), die etliche Zitate aus zeitgenössischen Zeitungen anführen, aus denen hervorgeht, dass die manipulative >Doppelzüngigkeit< der Nazis Anfang der 1930er Jahre durchaus auch im medialen Diskurs kritisch thematisiert wurde). Parteien, die Wähler*innen mit unterschiedlichen Interessen ansprechen wollen, agieren ähnlich ambivalent. Etliche Politiker [sic!] der FPÖ verhalten sich doppelzüngig, zumal wenn sie höhere politische Ämter anstreben. Ein klares Beispiel hierfür ist Norbert Hofer, der ehemalige Präsidentschaftskandidat der FPÖ. Hans-Henning Scharsach bringt Hofers Widersprüchlichkeit folgendermaßen auf den Punkt:

Er ist Mitglied einer antisemitischen Gemeinschaft, aber gegen Antisemitismus, er ist gleichzeitig für und gegen die österreichische Nation, für und gegen das Wiederbetätigungsgesetz, er schreibt nationalsozialistische Politikelemente in das Parteiprogramm, ist aber gegen Nationalsozialismus, er ist für und gegen 
die christliche Kirche, für und gegen Habsburg und den Adel, für und gegen die Identitären, für den kleinen Mann, aber gleichzeitig auch für Unternehmer und Millionäre und jedenfalls gegen Sozialgesetze, für und gegen Wissenschaft und Forschung, für und gegen den Tourismus. Diese Grundsatz(un)treue hat einen Namen. Man nennt sie Rechtspopulismus. (Scharsach 2017, S. 205)

Die Aufzählung von Gegensätzen lässt sich erweitern, denken wir etwa daran, dass Norbert Hofer einerseits Klimaschutz als wichtiges Anliegen der FPÖ erscheinen lässt, dass er als Verkehrsminister aber dafür verantwortlich zeichnete, auf manchen Autobahnabschnitten in Österreich das Tempolimit testweise auf $140 \mathrm{~km} / \mathrm{h}$ erhöht zu haben, und dass in seiner Zeit als Verkehrsminister für den Flughafen Wien-Schwechat der Bau einer dritten Piste genehmigt wurde - beides eindeutig klimaschädigende politische Entscheidungen.

Es sind Beispiele wie diese, die mich an der eher essayistisch-impressionistischen als empirisch belegten These Robert Pfallers, Thomas Bauers und Cornelia Koppetschs zweifeln lassen, dass wir es gegenwärtig mit einem Schwinden der Ambiguitätstoleranz und einer Tendenz zur Vereindeutigung der Welt und affektiven Entmischung zu tun haben (siehe Koppetsch 2019, S. 56-57, die sich auf Bauer 2018, S. 82-86 und Pfaller 2008 bezieht). Dieser pauschale Befund einer neuen Rigidität im Rechtspopulismus des globalen Zeitalters lässt sich mit Blick auf die FPÖ, aber auch auf andere rechtspopulistische Parteien, nicht wirklich bestätigen (und Bauers These von der Ambiguitätsfeindlichkeit des Kapitalismus ist grundsätzlich nicht haltbar). Zwar folgen Rechtspopulist*innen vielfach dem rhetorischen Prinzip der Komplexitätsreduktion, vor allem dann, wenn es um die Konstruktion von internen und externen >Feindbildern $<$, aber auch von >Freundbildern $<$ geht. Andererseits gehen sie in dem Bestreben, ihren Einfluss zu mehren, aber immer wieder strategisch Widersprüche ein, gestehen sich selbst also bewusst Mehrdeutigkeiten zu. Es liegt mithin eine rechtspopulistische Widerspruchsintoleranz gegenüber den anderen und eine Widerspruchstoleranz gegenüber sich selbst vor. Das »dynamische Paradox «, das sich in einer Kombination des Entweder-Oder mit dem Sowohl-alsauch äußert (Dietze 2018, S. 35), zeigt sich auch in den rechtspopulistischen Darstellungen der Geschlechterverhältnisse durch die FPÖ, die im folgenden Abschnitt fokussiert werden. Das Paradox ist laut Gabriele Dietze (2018, S. 36) sogar ein »konstituierendes Element für den derzeitigen historischen Erfolg des europäischen Rechtspopulismus $\ll{ }^{6}$

\section{Ambivalente rechtspopulistische Repräsentationen von Geschlechterverhältnissen in der FPÖ}

Anhand von acht Beispielen soll die kalkulierte Ambivalenz der FPÖ in der Darstellung der Beziehung zwischen den Geschlechtern betrachtet werden.

\footnotetext{
6 Siehe zu der von mir vertretenen These auch Olschanski (2017, S. 158-166), der ebenfalls eine »Hybridisierung der Rede« im Populismus beobachtet, die zu Doppeldeutigkeit führt.
} 
Das erste Beispiel ist dem FPÖ-Parteiprogramm aus dem Jahr 2011 entnommen. Dort ist im vierten Abschnitt über »Familie und Generationen« zu lesen:

Die Familie als Gemeinschaft von Mann und Frau mit gemeinsamen Kindern ist die natürliche Keimzelle und Klammer für eine funktionierende Gesellschaft und garantiert gemeinsam mit der Solidarität der Generationen unsere Zukunftsfähigkeit.

Wir bekennen uns zur Chancengleichheit von Männern und Frauen, zum gegenseitigen Respekt und zu einem gerechten Einkommen, unabhängig vom Geschlecht.

Die Bevorzugung eines Geschlechts zur Beseitigung tatsächlicher oder vermeintlicher Benachteiligungen wird von uns entschieden abgelehnt. Statistisch errechnete Ungleichheiten, die durch eine Vielzahl an Faktoren bedingt sind, können nicht durch Unrecht an einzelnen Menschen ausgeglichen werden. Daher sprechen wir uns gegen eine Quotenregelung oder das »Gender-Mainstreaming « aus. [...]

Wir bekennen uns zur Vorrangstellung der Ehe zwischen Mann und Frau als besondere Form des Schutzes des Kindeswohls. Nur die Partnerschaft von Mann und Frau ermöglicht unserer Gesellschaft Kinderreichtum. Ein eigenes Rechtsinstitut für gleichgeschlechtliche Beziehungen lehnen wir ab. [...]

Die Betreuung von Kindern in familiärer Geborgenheit wird von uns staatlichen Ersatzmaßnahmen vorgezogen. Unterstützungsleistungen dafür sind den Eltern bis zum Schuleintrittsalter zur Verfügung zu stellen. Gleichzeitig treten wir für ein breites Angebot an Kinderbetreuungsplätzen ein, um echte Wahlfreiheit zugunsten von Kindeswohl und beruflicher Entfaltung sicherzustellen. (FPÖ 2013, S. 115-116)

Hier wird von der FPÖ zunächst die der Fortpflanzung dienende Familie als soziale Einheit prioritär gesetzt und naturalisiert. Darauf folgt ein abstraktes symbolisches Bekenntnis zur Chancengleichheit von Männern und Frauen ${ }^{7}$, in dem die dritte von Josef Klein identifizierte Regel der kalkulierten Ambivalenz zur Anwendung kommt. In Widerspruch zu diesem allgemeinen Credo steht die unmittelbar anschließende emphatische Ablehnung von Maßnahmen einer Affirmative Action, also einer temporären positiven Diskriminierung von Frauen, die zu mehr Gleichheit führen könnte. Diese Ablehnung von Quotenregelungen und Strategien zur Förderung der Gleichstellung der Geschlechter nimmt im Parteiprogramm mehr als doppelt so viel Textraum ein als das allgemeine Bekenntnis zur Chancengleichheit. Damit ist eine Gewichtung vorgenommen. Die Ablehnung wird individualistisch begründet (»Unrecht an einzelnen Menschen«). Die realen asymmetrischen Geschlechterverhältnisse zwischen Frauen und Männern, die in vielen gesellschaftlichen Bereichen noch immer durch systematische Ungleichbehandlungen gekennzeichnet sind, blendet die FPÖ aus. Abgelehnt wird im Parteiprogramm der FPÖ dann auch das Rechtsinstitut

\footnotetext{
7 Fünf Mal sind in der ersten Hälfte des vierten Textabschnitts des Parteiprogramms Frauen und Männer erwähnt, immer in der gleichen Reihenfolge, die zuerst die Männer und dann die Frauen erwähnt, und in vier der fünf Nennungen wird der pauschale Kollektivsingular in der Doppelform »Mann und Frau« gebraucht.
} 
der Ehe für homosexuelle Männer und lesbische Frauen. ${ }^{8}$ Ambivalent fällt auch die Textpassage zur Kinderbetreuung aus. Einerseits finden wir darin den Prioritätentopos, mit dem sich die freiheitliche Wir-Gruppe für eine Betreuung der Kinder zuhause ausspricht (dass - vielfach aus Gründen der gesellschaftlichen Ungleichbehandlung - in der Regel Frauen daheim bei den Kindern bleiben, ist hier ungesagt). Andererseits wird ein breites Kinderbetreuungsangebot gefordert, das »echte Wahlfreiheit « und berufliche Entfaltung (von wem, das wird nicht mitgeteilt) ermöglichen solle. Es wird hier also beides - familiäre und außerfamiliäre Betreuung - angeführt, um es unterschiedlichen Gruppen von Wähler*innen recht zu machen. Die Formulierung lässt aber keinen Zweifel daran, dass außerfamiliäre Kinderbetreuung für die FPÖ nur eine »Ersatzmaßnahme « ist.

Dass im Parteiprogramm der FPÖ kalkuliert ambivalente Darstellungen von Geschlechterverhältnissen vorkommen, ist erwartbar, handelt es sich doch um eine Textart, die gerne strategisch mit Ambivalenzen gespickt wird. Betrachten wir eine weitere programmatische Textart als zweites Beispiel. Kalkulierte Ambivalenzen finden sich auch im Wahlprogramm der FPÖ aus dem Jahr 2017. Der schillernde Text ist sehr aufschlussreich. Er gehorcht insgesamt dem Prinzip der kalkulierten Ambivalenz, weil das Thema der Gerechtigkeit (»Fairness «) den roten Faden des Wahlprogramms darstellt, der vor allem auch SPÖ-Wähler*innen ansprechen soll. Das Wahlprogramm besteht aus einem Vorwort des Parteichefs Heinz-Christian Strache und 25 Abschnitten mit insgesamt »100 FPÖ-Forderungen zur Beseitigung der Fairness-Krise«. Der elfte Abschnitt ist mit der Forderung »Unsere Frauen gleichberechtigen und sie vor Diskriminierungen schützen« überschrieben. Im Fließtext lesen wir dann (Beispiel 2):

Frauen verdienen bei gleicher Arbeit immer noch weniger als Männer. Dieser Missstand muss beseitigt werden. Es kann nur gelten: Gleicher Lohn für gleiche Arbeit. [...]

Neben der Verbesserung der Vereinbarkeit von Beruf und Familie sind qualitätsvolle Teilzeitarbeitsplätze sowie gute Möglichkeiten zum beruflichen Wiedereinstieg zu schaffen. Die FPÖ steht für eine gelebte Gleichberechtigung der Geschlechter. Das bedeutet aber keine Gleichmacherei von Mann und Frau. Deshalb sprechen wir uns gegen scheinheilige Alibiaktionen in der Frauenförderung aus, die nicht geeignet sind, die Stellung der Frau tatsächlich aufzuwerten. Wir akzeptieren keine Unterdrückung von Frauen und lehnen daher Zwangsheirat oder Kopftuchzwang strikt ab. (FPÖ 2017, S. 24, links)

Auch wenn formal schnell erkennbar ist, dass alle 25 Abschnitte das gleiche Format und die gleiche Gliederung aufweisen und mit dem possessiven personalen Deiktikum unser beginnen, so ist doch sehr bemerkenswert, dass die Nomination

\footnotetext{
${ }^{8}$ Eine vergleichbare konservative Position zu Ehe und Familie findet sich im Parteiprogramm der AfD (2016, S. 78, 80). Dem steht die mediale Selbstdarstellung der Ko-Vorsitzenden der Bundestagsfraktion der AfD, Alice Weidel, gegenüber, die sich 2017 als lesbisch lebende Mutter outete, die mit ihrer aus Sri Lanka stammenden Lebensgefährtin zwei Söhne großzieht. Somit oszilliert auch die AfD kalkuliert ambivalent zwischen konservativer programmatischer Geschlechterpolitik und progressiver Geschlechterperformance einer Spitzenpolitikerin (siehe hierzu Dietze 2018, S. 40).
} 
unsere Frauen im Titel des Abschnitts ein restriktives possessivisches Verhältnis zwischen der Wir-Gruppe und der Gruppe der Frauen etabliert. Egal, ob sich das ambige unser eher auf ein Partei-Wir oder ein österreichisches Wir bezieht, es ist ein androzentrisches Wir, also ein frauenexklusives Männer-Wir. Damit outet sich die FPÖ als Männerpartei, die Frauen zum Objekt macht, dem etwas Positives widerfahren soll, nämlich dass es gleichberechtigt und vor Diskriminierung geschützt wird. Bedeutsam ist auch die Präsupposition, die in der Forderung steckt, nämlich dass »unsere Frauen« zum Sprech- und Schreibzeitpunkt, also 2017, noch nicht gleichberechtigt sind.

Die fehlende Gleichberechtigung wird am Anfang des Abschnitts konkretisiert. Es wird kritisiert, dass für gleiche Arbeit ungleicher Lohn bezahlt werde, also ein geschlechtstypisches Lohngefälle bestehe. Für die Beseitigung dieser Ungerechtigkeit spricht sich die FPÖ aus. Allgemein fordert sie auch die Verbesserung der Vereinbarkeit von Beruf und Familie. An der Problematik, dass es primär Frauen sind, die nur einer Teilzeiterwerbstätigkeit nachgehen (können), da sie den Großteil der Kinderbetreuung in der Familie übernehmen, will die FPÖ dem Wahlprogramm zufolge aber nichts ändern, begrüßt sie es doch, wenn »eine Mutter [...] bei ihren Kindern zu Hause bleiben will« (FPÖ 2017, S. 24, links; man notiere den Plural, der die Erwartung einer Geburtenrate von mindestens 2 ausdrückt).

Zwar hat sich der ehemalige FPÖ-Chef Heinz-Christian Strache zudem für einen >Papamonat< stark gemacht und diesen im Januar 2019 selbst auch als Vizekanzler, Sportminister und FPÖ-Chef in Anspruch genommen. Allerdings ist hierzu zweierlei anzumerken. Erstens: Was ist schon ein Monat im Vergleich zu der jahrzehntelangen Betreuungstätigkeit, die leider immer noch zum Großteil von Frauen geleistet wird, um Kinder auf ihrem Weg zur Volljährigkeit fürsorglich und zumeist unbezahlt zu begleiten? Nimmt sich da ein >Papamonat< nicht primär wie Symbolpolitik aus? Zweitens ist Strache unglaubwürdig, weil seine verbale Selbstdarstellung in starkem Gegensatz zu seinem Handeln als frisch gebackener Vater steht. Dazu äußert sich die Journalistin Nina Horaczek in der österreichischen Wochenzeitschrift Falter am 20. Februar 2019 in einem kurzen Artikel mit dem elliptischen Titel »Wie gedruckt«, der unschwer zur Redewendung »Er lügt wie gedruckt« ergänzt werden kann. Horaczek stellt der Behauptung Straches, dass ihn der Papamonat entschleunigt habe, eine lange Liste leicht recherchierbarer Aktivitäten Straches während seiner angeblichen Babypause gegenüber. Die Liste vermittelt den Eindruck, dass Strache in seinem >Papamonat< viele Tage kaum so bei seinem Kind war, wie es das Modell des $>$ Papamonats< eigentlich vorsehen würde. ${ }^{9}$

Kehren wir zurück zum FPÖ-Wahlprogramm und dem Abschnitt über die Frauenpolitik. Im letzten Teil des obigen Zitats wird die »Gleichberechtigung « als Hochwertwort affirmiert und der »Gleichmacherei von Mann und Frau« (man notiere die Kollektivsingulare) ablehnend gegenübergestellt. Das komplexe Nomen Gleichmacherei denotiert die deontisch unerwünschte Einebnung gegebener Differenzen und ist negativ konnotiert, also ein Unwertwort. Die Formulierung enthält einen impliziten Differenztopos, der besagt, dass Verschiedenes nicht gleich, sondern verschieden behandelt werden solle. Der Gleichheitstopos, der Frauen und Männern

\footnotetext{
${ }^{9}$ Für den Hinweis auf den Artikel bin ich Verena Krausneker dankbar.
} 
die gleichen Rechte einräumt, steht mit dem Differenztopos zumindest teilweise in Konflikt. Das zeigt sich dort, wo im Zitat Frauenförderung, die zu mehr Gleichberechtigung führen soll, als scheinheilige Alibiaktion abgetan wird, aber auch auf der rechten Seite des gleichen Textabschnitts. Dort heißt es: Unfair sei, »[d]ass durch die Einwanderung von Menschen aus patriarchalen Kulturen eine neue Frauendiskriminierung stattfindet « und »[d]ass Alibimaßnahmen wie das Binnen-I und die Nennung von Töchtern in der Bundeshymne von den wirklichen Problemen der Frauen ablenken«. Die FPÖ fordert »tatsächliche Gleichberechtigung statt heuchlerischer Gleichmacherei« und »Nulltoleranz gegenüber jeder Form von Gewalt und Unterdrückung von Frauen und Kindern« (FPÖ 2017, S. 24, rechts). Das heißt: Das Recht von Frauen, im Sprachgebrauch gleichberechtigt behandelt zu werden, wird ebenso als Alibimaßnahme und heuchlerische Gleichmacherei abgelehnt wie die Nennung von Töchtern zusätzlich zu den Söhnen in der österreichischen Bundeshymne. Im Bereich des Sprachgebrauchs sollen Frauen somit nicht das gleiche Recht erhalten wie Männer, symmetrisch mitgenannt zu werden. Eine schlüssige Begründung für diese diskriminierende Ungleichbehandlung wird nicht gegeben, eine trugschlüssige allerdings schon - in Form eines Prioritätentopos, der die Frage des geschlechtergerechten Sprachgebrauchs zu etwas Unwichtigem herabstuft, das von den wirklichen Problemen der Frauen wegführe. Was an den schon zitierten Stellen aus dem Wahlprogramm hinsichtlich der Repräsentation der Geschlechterverhältnisse zudem auffällt, das ist die Kritik an der Diskriminierung von Frauen durch Männer aus patriarchalen Kulturen, die nach Österreich eingewandert sind. Dieser Sexismus bzw. Genderismus, etwa in Form von Kopftuchzwang und Zwangsverheiratung und in Form von tätlicher Gewalt gegen Frauen, wird strikt zurückgewiesen. Bestimmte Formen der Diskriminierung von Frauen, die von Österreichern und der FPÖ vorgenommen werden, stellen dagegen einen blinden Fleck dar. Die FPÖ misst hier aus strategischen Gründen mit zweierlei Maß, verhält sich kalkuliert ambivalent: Frauendiskriminierung von Zugewanderten wird aus strategischen und insbesondere auch fremdenfeindlichen Gründen verurteilt und tendenziell ethnisiert, die eigene Geschlechterdiskriminierung wird dagegen weitgehend übergangen oder gerechtfertigt. Diese Geschlechterdiskriminierung durch die FPÖ zeigt sich in der hierarchisierenden Naturalisierung binärer Geschlechterdifferenzen und eines patriarchalen Familienbildes, ${ }^{10}$ in der Ablehnung vieler Maßnahmen zur Frauenförderung und von Gender-Mainstreaming, in der Ablehnung von Geschlechtervielfalt und in der Ablehnung eines geschlechtergerechten Sprachgebrauchs.

\footnotetext{
10 Siehe dazu die in Abschnitt 1 erwähnten Beispiele, aber auch das von Norbert Hofer 2013 herausgegebene Buch Michael Howanietz' mit dem Titel »Für ein freies Österreich«. Das Buch, zu dem sowohl Strache als auch Hofer ein wohlwollendes Vorwort beigetragen haben, strotzt auf den Seiten 32, 110, 112, 113, 118, 119 vor rückwärtsgewandten Geschlechterstereotypen, die von Emanzipation und Gleichberechtigung meilenweit entfernt sind. Eine Textstelle sei stellvertretend zitiert: »Der vom Thron des Familienoberhaupts gestoßene Mann sehnt sich unverändert nach einer Partnerin, die, trotz hipper den-Mädelsgehört-die Welt-Journale, in häuslichen Kategorien zu denken imstande ist, deren Brutpflegetrieb auferlegte Selbstverwirklichungsambitionen überragt. Die von feministischem Dekonstruktionsehrgeiz zur selbstverwirklichungsverpflichteten Geburtsscheinmutter umdefinierte Frau sehnt sich nach einem ganzen Kerl, der ihr alle die emotionalen und ökonomischen Sicherheiten gibt, die eine junge Mutter braucht, um sich mit weitgehend sorgloser Hingabe dem Nachwuchs zuwenden zu können.« (Howanietz 2013, S. 32). Siehe auch Falter/Stern (2019 S. 189-190).
} 
Abb. 3 Beispiel 3: FPÖ-Polemik gegen das Binnen-I als Ausdruck von >Genderwahn< (http://facebook.com/strache; das Facebook-Posting wurde zuletzt abgerufen am 27.2.2019)

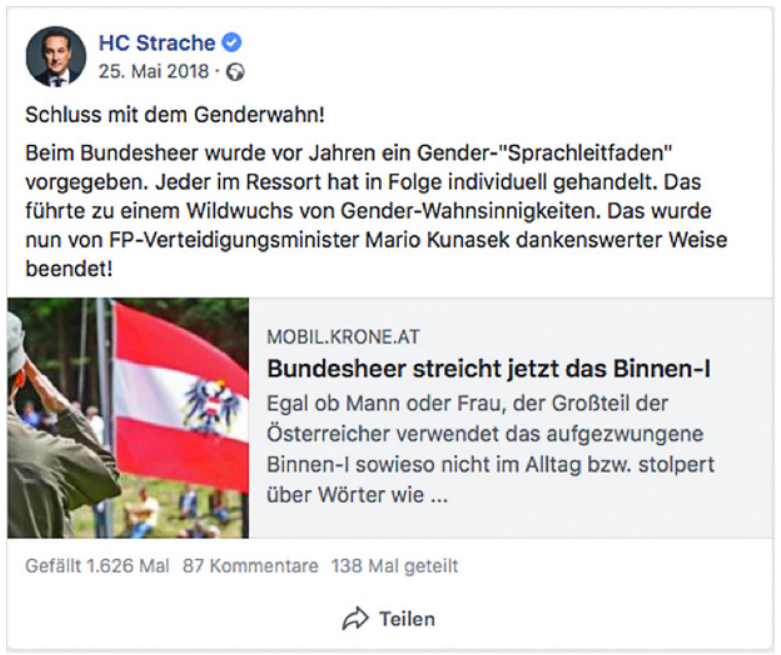

Für die Linguistik besonders interessant ist die vehemente Zurückweisung des Binnen-I. Das in den 1990er Jahren als Kurzform für sprachliche Gleichbehandlung von Frauen und Männern in Umlauf gekommene Graphem wird, wie Beispiel 3 (siehe Abb. 3) illustriert, von Heinz-Christian Strache als Ausdruck des >Genderwahns interpretiert:

Hier fordert Strache die Beendigung des angeblichen >Genderwahns<. Mit der Metapher des Wahns wird das Binnen-I als pathologische Manifestation diskreditiert. Dankend bezieht sich Strache auf einen in der Kronenzeitung erwähnten Erlass des ehemaligen FPÖ-Verteidigungsministers Mario Kunasek vom 23. April 2018, der angebliche Erlässe aus dem Jahr 2001, die im Verteidigungsressort das Binnen-I und die Beidnennung von Frauen und Männern vorschreiben würden, außer Kraft setze. Ironischerweise gab es derartige Erlässe zum Binnen-I und der Beidnennung allerdings nie, liegt hier also primär Symbolpolitik vor. Eine vergleichbare Symbolpolitik betrieben auch freiheitliche Politikerinnen, als sie anlässlich des Weltfrauentages 2016 eine Pressekonferenz abhielten, die sich gegen die sogenannte $>$ Genderideologie< und gegen den sogenannten >Genderwahn< richtete, der gestoppt werden müsse. Auch damals griff Strache den antifeministischen FPÖ-Aktivismus auf. Am 8. März 2016 verbreitete er ein Video zu dieser Pressekonferenz auf seiner Facebook-Seite und sprach sich gegen den angeblichen >Genderwahn < aus, der den Frauen gar nichts nutze.

Die Tendenz zur »Ethnisierung von Sexismus« (vgl. zu diesem Konzept M. Jäger (1996, S. 155-293) bzw. zu dem von Gabriele Dietze sogenannten »Islam-Sexualitäts-Emanzipations-Nexus« (Dietze 2018, S. 36-38) sei mit dem vierten Beispiel illustriert (siehe Abb. 4). ${ }^{11}$ Im Oktober 2018 brachten die »Freiheitlichen Arbeitnehmer« ein bebildertes Werbekonstrukt in Umlauf, das eine leicht lächelnde, Kopftuch

11 Auch Marine Le Pen, die Chefin des Front National, betreibt die Ethnisierung von Frauendiskriminierung. Als weibliche Führungsfigur gibt sie sich machtbewusst emanzipiert. Einen Femonationalismus vertritt sie dort, wo sie 2016 davor warnt, dass die Flüchtlingskrise der Anfang vom Ende der Frauenrech- 
Abb. 4 Beispiel 4: Symbolpolitischer »xenophober « Kampf gegen den vermeintlich spezifisch ethnischen Sexismus (SOS Mitmensch 2018, S. 27)

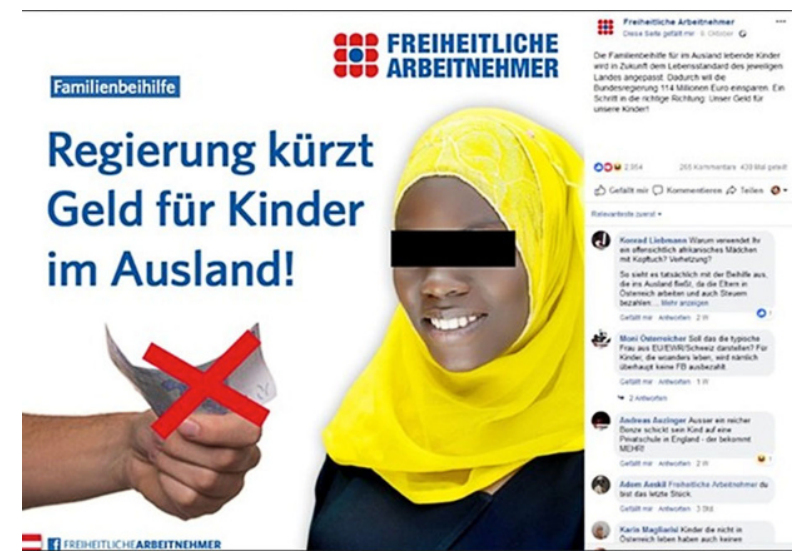

tragende afrikanische Muslima zeigen soll, der sich eine Hand mit einem durchgestrichenen Geldschein entgegengestreckt. Überschrieben ist das Bild mit dem Ausrufesatz: »Regierung kürzt Geld für Kinder im Ausland!« Das visuelle Stereotyp operiert mit dem Kollektivsymbol des Kopftuchs, das für die kulturelle Andersartigkeit und muslimische Unterdrückung von Frauen stehen soll. Die implizite Botschaft des Plakates lautet: Wir, die ÖVP-FPÖ-Regierung, fördern ausländische sexistische Kulturen nicht, die Frauen durch Kopftuchzwang unterdrücken und unser System der Familienbeihilfe durch ihren Kinderreichtum ausnutzen. Wie sehr dieses Plakat darauf abzielt, diskriminierende Vorurteile zu schüren, hat die NGO SOS Mitmensch deutlich gemacht, als sie darauf hinwies, dass 90 Prozent der ausländischen Bezieher*innen von Familienbeihilfe aus osteuropäischen Nachbarländern Österreichs stammen und dass außerhalb der EU und EWR gar keine Familienbeihilfe ausgezahlt werde. Insofern wurde mit dem Plakat aus fremdenfeindlichen Gründen vorsätzlich gelogen.

Strategische Kritik an Sexismus findet sich auch in Beispiel 5. Dieses Mal richtet sich die Verurteilung von Sexismus gegen den jahrzehntelangen FPÖ-Chef Jörg Haider, der im April 2005 die FPÖ gespalten und eine eigene Partei, das Bündnis Zukunft Österreich (BZÖ), gegründet hatte. Das Büro des neuen FPÖ-Obmanns Heinz-Christian Strache kritisierte in der Presseaussendung vom 24. Juni 2006 »Widerliche sexistische Pseudopointen Jörg Haiders« - so der Titel der Aussendung. Darin hieß es:

Die gewesene Oberorange Jörg Haider hat die sozialdemokratische Bundesgeschäftsführerin Doris Bures gestern als Frau, »die selbst der Bulle von Tölz ablehnen würde «, bezeichnet.

Bei aller Schärfe, die in der tagespolitischen Auseinandersetzung herrscht und oft auch geboten ist, und unter Berücksichtigung aller Abgründe, die uns von der SPÖ trennen: Aber diese Aussage ist absolut letztklassig. Offenbar hat Jörg 
Haider die einfachst strukturierte Gedankenwelt seines ehemaligen Kofferträgers und nunmehrigen Chefs bereits voll und ganz in sich aufgesaugt.

Wir sind froh, dass jemand, der derartig widerliche sexistische Pseudopointen schleudert, nicht mehr unserer Partei angehört. Und wir sind auch froh, dass Leute, die solchen Aussagen noch mit begeistertem Johlen applaudieren, nicht mehr unserer Partei angehören. [...]

Den Kärntner Landeshauptmann fordern wir auf, sich in aller gebotenen Form bei Doris Bures zu entschuldigen. (Büro HC Strache 2006, https://www.ots. at/presseaussendung/OTS_20060624_OTS0003/fpd-widerliche-sexistischepseudopointen-joerg-haiders)

Die Sexismus-Kritik der FPÖ beginnt hier mit einer hierarchisierenden metaphorischen Benamsung Haiders als oberste Zitrusfrucht. Die verballhornende Benamsung »die Oberorange« ergibt sich in Anspielung auf die Parteifarbe des BZÖ, also Orange, in Verbindung mit der Substitution des maskulinen durch den femininen Artikel. Die ironisierende Verspottung wird hier Haider und sonst - z. B. in zwei Presseaussendungen Herbert Kickls - noch Peter Westenthaler zuteil, der am 23. Juni 2006, einen Tag vor der hier zitierten Presseaussendung, zum neuen Bündnisobmann des BZÖ gewählt worden war. Westenthaler wird im zweiten Absatz der FPÖ-Aussendung zum ehemaligen Kofferträger Haiders herabgesetzt, der in einer »einfachst strukturierten Gedankenwelt« lebe, die Haider wohl völlig übernommen habe, als er die SPÖ-Politikerin sexistisch attackierte.

Die Glaubwürdigkeit der Sexismus-Kritik wird zuallererst dadurch untergraben, dass Daniela Plachutta, die von Strache geschiedene Frau, wenige Wochen vor der Presseaussendung des Strache-Büros, nämlich am 7. Juni 2006, in der österreichischen Zeitschrift News mit der Aussage zitiert wird, dass sie sich in den letzten Jahren ihrer Ehe mit Heinz-Christian Strache als unnötiger Putz- und Hausfetzen gefühlt habe. Diese Darstellung lässt den damaligen FPÖ-Chef Strache nicht unbedingt als glaubwürdigen Kritiker von Haiders frauenfeindlicher Äußerung erscheinen.

Und auch Beispiel 6 (siehe Abb. 5), ein Kommentar, den Strache am 31. Januar 2013 auf seiner Facebook-Seite veröffentlichte, trägt nicht dazu bei, Strache als überzeugenden Kämpfer gegen Frauendiskriminierung dastehen zu lassen: ${ }^{12}$

Auch wenn Straches situational getriggerter Kommentar »Heute am Jägerball mit meinem Schatz.... Viele Jäger und Hasen ;))) >auf, auf, ihr Hasen, hört ihr nicht die Jäger blasen! $<$ ))) « scherzhaft war und entsprechend markiert ist, aktiviert der Kalauer doch einen animalisierenden Deutungsrahmen, der die Anbahnung zwischengeschlechtlicher und sexueller Beziehungen als Jagd metaphorisiert, bei der Männer Frauen wie Hasen jagen, die vor den Männern fliehen. Die Metapher überträgt nicht nur Merkmale von männlicher Überlegenheit und weiblicher Schwäche

\footnotetext{
12 Straches Glaubwürdigkeit wird etwa auch dadurch untergraben, dass er am 10. Februar 2016 in seiner Aschermittwochsrede die damalige ÖVP-Innenministerin Johanna Mikl-Leitner unter anderem mit den Worten kritisierte, auch »keine Schönheitskönigin« zu sein (siehe dazu Olschanski 2017, S. 136). Andererseits wird Strache Ende Oktober 2019 der männlichen Führungsriege der FPÖ Frauenfeindlichkeit vorwerfen, weil sie seine Ehefrau Philippa Strache - wegen parteischädigenden Verhaltens - aus der FPÖ ausschloss.
} 
Abb. 5 Beispiel 6: Metaphorisierung zwischengeschlechtlicher und sexueller Beziehungen als Jagd (https://www. vol.at/sexismus-eklat-fp-chefstrache-erhitzt-mit-posting-diegemueter/3475588 2013)
Q43 KOMmentare $\quad 31.01 .2013$ 14:09 (Akt. 31.01.2013 16:02)

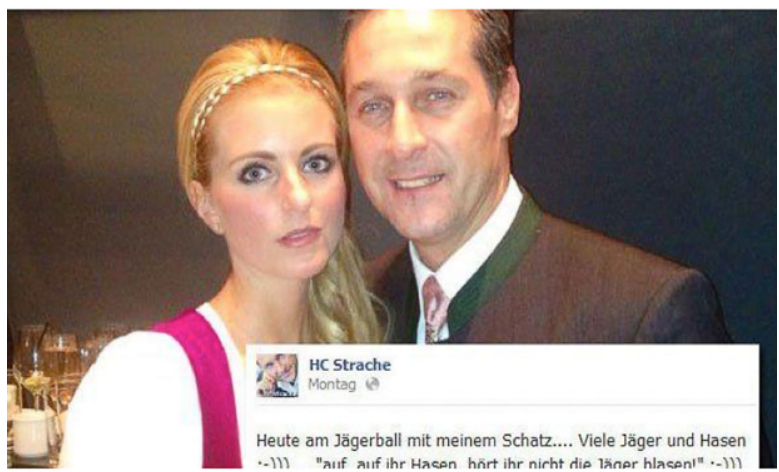
Sexismus oder nicht? Strache-Posting spaltet Meinungen. cscreenshot/ Facebook HC Strache

Mit einem Posting auf Facebook hat FP-Chef H.C. Strache die aktuelle Sexismus-Debatte noch angeheizt.

sowie weiblicher Flucht vor männlicher Bedrohung, sondern darüber hinaus auch hochproblematische Phantasien von sexueller Gewalt.

Eine Doppel-Metapher steht im Zentrum des siebten Beispiels, nämlich die Metapher des Herrn im eigenen Haus. Diese androzentrische Gebäudemetapher hat Strache zwischen 2005 und 2014 immer wieder verwendet, um entweder in xenophober Absicht vor einer angeblichen Bedrohung der Minorisierung und Fremdbestimmung der »Österreicher « im eigenen Land zu warnen (siehe eine entsprechende Presseaussendung Straches vom 24. Juni 2006) oder um in EU-feindlicher Absicht gegen die österreichische Abhängigkeit von der EU im Bereich der Gesetzgebung zu agitieren. Letzteres ist in einem Posting auf Straches Facebook-Seite vom 28. April 2014 der Fall (Abb. 6).

Abb. 6 Beispiel 7: EU-kritische androzentrische Metapher des Herren im eigenen Haus (http://facebook.com/strache; das Facebook-Posting wurde zuletzt abgerufen am 27.2.2019)
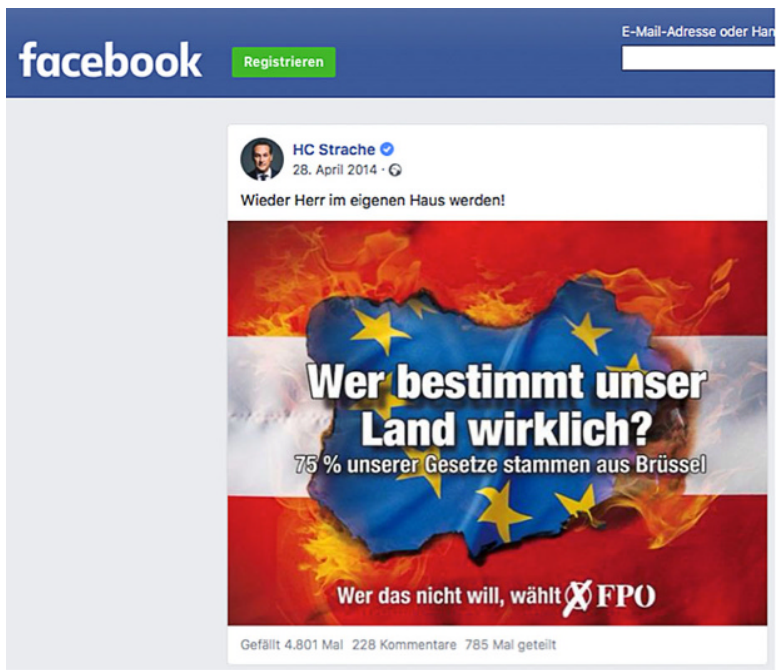
Auch diese Metapher steht für eine männliche Perspektive der Dominanz, aber gleichzeitig auch für einen nationalen oder staatlichen Abgrenzungs- und Autonomieanspruch, ist die Hausmetapher doch eine Container-Metapher, mit der nach außen Abgrenzung und nach innen Schutz suggeriert werden soll.

Betrachten wir ein letztes, achtes Beispiel, um unsere Blütenlese zur kalkulierten Ambivalenz der FPÖ abzurunden. Anlässlich des internationalen Frauentags am 8. März 2018, also auf den Tag genau zwei Jahre, nachdem Strache sich gegen Binnen-I und Genderwahn ausgesprochen hat, wird der nunmehrige Vize-Kanzler von der Journalistin Martina Madner in der Wiener Zeitung gefragt, ob er Feminist sei. Die Antwort Straches fällt einmal mehr ambivalent aus (Beispiel 8):

»Wenn Feminismus bedeutet, für die Gleichberechtigung von Mann und Frau einzutreten, dann bin ich Feminist. Wenn Feminismus hingegen bedeutet, die biologischen Unterschiede von Mann und Frau zu leugnen und künstlich hundert verschiedene Geschlechter postulieren zu wollen, bin ich kein Feminist.« In der Sache aber gibt er ein Bekenntnis ab: »Die Gleichberechtigung der Geschlechter ist für mich eine der wichtigsten Errungenschaften der Menschheitsgeschichte.«

Auch für den Vizekanzler ist noch nicht alles in Butter: »Es gibt hier noch viel zu tun.« Zu gleichen Rechten und Pflichten, Chancengleichheit, gibt es vom Vizekanzler ein Ja; zu Gleichmacherei aber ein Nein. Er betont die »Verschiedenheit« beider Geschlechter, wie im Regierungsprogramm. (Madner 2018; https://www.wienerzeitung.at/nachrichten/politik/oesterreich/951344Sind-Sie-Feminist.html?em_no_split=1)

Strache oszilliert hier zwischen einem Gleichheits- und Differenztopos. Er bekennt sich zur Gleichberechtigung von Frauen und Männern, betont gleichzeitig aber die biologische Differenz dieser beiden Geschlechter. Die Realität der Geschlechtervielfalt jenseits der Binarität von männlich und weiblich lehnt er ab. Geschlecht sieht er als rein biologische Kategorie an. Er naturalisiert sie voll und ganz. Widersprüchlich setzt er im Interview fort. Die Gleichberechtigung der Geschlechter (gemeint sind nur Frauen und Männer) sei für Strache eine der wichtigsten Errungenschaften der Menschheitsgeschichte. Mit dieser Assertion suggeriert er, dass die Gleichberechtigung von Männern und Frauen schon erreicht sei. Dann gesteht er aber ein, dass es noch viel zu tun gebe (insofern scheint die Gleichberechtigung doch noch nicht erreicht zu sein). Ganz auf der Linie des Parteiprogramms von 2011 bekennt sich Strache zu gleichen Rechten und Pflichten und zur Chancengleichheit für Frauen und Männer, er weist aber gleichzeitig die >Gleichmacherei< zurück und hebt die Verschiedenheit von Männern und Frauen hervor.

Diese schon im Parteiprogramm (Beispiel 1) beobachtete Ambivalenz löst sich nicht auf, sondern dient dazu, unterschiedliche Gruppen von Wähler*innen gleichermaßen zufriedenzustellen, darunter eben auch mehr Frauen als bisher. Während die Affirmation der Gleichberechtigung vor allem ein Signal an egalitär gesinnte Frauen ist, stellt das Hochhalten biologistisch gefasster asymmetrischer Geschlechterrollen eine Kontinuitätsbotschaft für bisherige Anhänger*innen der FPÖ dar. Es scheint, als wäre die Ambivalenz und Widersprüchlichkeit in der Geschlechterpolitik der rechtspopulistischen FPÖ in der Formel >Gleichberechtigung ja, aber keine Gleichmache- 
rei< kodiert auf den Punkt gebracht. Hinter der FPÖ-Kritik an der >Gleichmacherei< verbirgt sich primär die Ablehnung vieler politischer Maßnahmen, die tatsächlich für mehr Gleichberechtigung von Frauen und Männern sorgen könn(t)en.

\section{Fazit}

Fassen wir zusammen. Strategisch ambivalentes Formulieren ist in der Politik gebräuchlich. Auch wenn im Vorangegangenen der Blick auf den rechten politischen Rand und das Feld der Geschlechterpolitik fiel, finden sich kalkulierte Ambivalenzen in allen Parteien und vielen Politikfeldern. Es gibt Kommunikationsformate, in denen das Prinzip der kalkulierten Ambivalenz besonders gerne angewandt wird. Prädestiniert für sie sind Parteiprogramme, Kommunikationstypen der politischen Werbung, konsensorientierte identitätsstiftende Kommunikationstypen, die politische Kontinuität und Diskontinuität artikulieren (wie z. B. Gedenkreden), diplomatische Textund Diskursarten sowie halbherzige Entschuldigungen.

Die Gründe für und Funktionen von kalkulierter Ambivalenz sind vielfältig: (1) Oft soll die Vereinbarkeit von Unvereinbarem suggeriert, eine harmonisierende Vereinigung von widerstreitenden Interessen erreicht werden. (2) Oft geht sie aus der Mitwirkung mehrerer Autor*innen, die unterschiedliche Ansichten vertreten sowie Interessen und Ziele verfolgen, hervor. (3) Ein starkes Motiv für sie ist die Mehrfachadressierung von Gruppen mit unterschiedlichen Ideologien, Weltanschauungen, ethischen Werthaltungen. (4) Strategisch ambivalent verhält sich auch, wer angesichts einer aktuellen Veränderung und Diskontinuität Kontinuität suggerieren will. (5) Die Vermeidung eindeutiger Positionierungen oder Entscheidungen führt ebenfalls zu Ambivalenz. (6) Wer sich nur augenzwinkernd und halbherzig distanzieren oder entschuldigen will, formuliert ebenfalls oft ambivalent. (7) Und wer einen Tabubruch vollziehen will, ohne zur Rechenschaft gezogen werden zu können, wendet auch das rhetorische Prinzip der kalkulierten Ambivalenz an.

In rechtspopulistischen Repräsentationen von Geschlechterverhältnissen manifestiert sich kalkulierte Ambivalenz vor allem dort, wo einerseits ein allgemeines und abstraktes Bekenntnis zur Gleichberechtigung von Frauen und Männern abgegeben wird und andererseits ein feindliches Bild von Feminismus und angeblichem »Genderwahn « gezeichnet wird, während der Antifeminismus und Genderismus in den eigenen Reihen gleichzeitig überspielt, relativiert oder - naturalisierend - gerechtfertigt wird und während die Realität der Geschlechtervielfalt nicht anerkannt wird. Kalkulierte Ambivalenz zeigt sich besonders auch dort, wo die Geschlechterdiskriminierung der anderen lautstark kritisiert und die eigene Geschlechterdiskriminierung ausgeblendet wird. Dies ist vor allem dann der Fall, wenn sich der Diskurs über Migration und Fremde mit dem Diskurs über Geschlecht verschränkt, um eine Ethnisierung von Sexismus zu betreiben. Es wird im politischen Umgang mit Geschlechterverhältnissen von der FPÖ, aber auch von anderen rechtspopulistischen Parteien, also gerne mit zweierlei Maß gemessen. Die rechtspopulistische Rhetorik folgt mithin gleichzeitig dem Prinzip der binären Komplexitätsreduktion und dem Prinzip der kalkulierten Ambivalenz. Die strategische und paradoxale Verknüpfung des Entweder-oder mit dem Sowohl-als-auch scheint für die Wahlerfolge 
rechtspopulistischer Parteien in den letzten Jahren und Jahrzehnten maßgeblich mitverantwortlich zu sein (Dietze 2018, S. 35). Eine zu starke Vereindeutigung würde in den gegenwärtigen Zeiten des raschen sozialen Wandels nämlich nur die Stammwähler*innen ansprechen.

Vergleicht man FPÖ und AfD, stellt sich der Eindruck ein, dass die FPÖ in der Repräsentation von Geschlechterverhältnissen in den letzten Jahren durch mehr kalkulierte Ambivalenz aufgefallen ist als die AfD. Es steht zu vermuten, dass dies auch mit dem Wechsel der FPÖ in die Regierung zu tun hat. Insofern lässt sich grundsätzlich fragen, ob Regierungspopulismus systembedingt stärker durch kalkulierte Ambivalenz gekennzeichnet ist als oppositioneller Rechtspopulismus. Bei der Beantwortung dieser Frage in zukünftigen politolinguistischen Untersuchungen ist differenzierend zu berücksichtigen, dass in Gesellschaften mit sehr patriarchalen bzw. genderistischen Traditionen wie Italien Geschlechterdiskriminierung auch im Regierungspopulismus stark präsent ist. Auf jeden Fall zeigt sich kalkulierte Ambivalenz sowohl im politischen Prozess des rechtspopulistischen Kampfes um Einfluss, also in der Politics, als auch im Bereich des rechtspopulistischen Regierungshandelns, also in der Policy, als strategisches Moment.

Kalkulierte Ambivalenz, die auf allzu offensichtlichen Unvereinbarkeiten und Widersprüchen beruht, führt allerdings zum Verlust der Glaubwürdigkeit. Die Analyse der genderbezogenen kalkulierten Ambivalenzen bietet daher die Möglichkeit einer antipopulistischen Kritik. Sie kann zeigen, dass das rechtspopulistische Bekenntnis zur Gleichberechtigung - wegen der fortdauernden Geschlechterdiskriminierung in den eigenen Reihen - oft ein sehr halbherziges oder gar verlogenes, zumindest jedoch unglaubwürdiges Bekenntnis ist und dass die Kritik an islamischem Sexismus bzw. Genderismus stark strategisch und mehr »xenophob« als aufklärerisch, emanzipatorisch oder feministisch motiviert ist.

Wer antipopulistische Kritik übt, sollte berücksichtigen, dass Geschlechterdiskriminierung ein systemisches Problem aller patriarchal geprägten Gesellschaften und Religionen darstellt, also etwa auch der christlichen Religion, und dass die größte Gefahr für sexuelle Übergriffe immer noch in den »eigenen vier Wänden« besteht. Kritiker*innen des Rechtspopulismus sollten auch bedenken, dass Rechtspopulist*innen ernst zu nehmende Probleme der Geschlechterdiskriminierung durch androkratisch geprägte Migrant*innen ansprechen, die auch Antipopulist*innen aufgreifen müssen, wollen sie den Zulauf zu rechtspopulistischen Parteien wieder verringern. Schließlich gilt es, sich vor Augen zu halten, dass Geschlechterverhältnisse historisch veränderbar sind, dass die (rechtliche) Gleichstellung in modernen europäischen Demokratien, wo sie gegeben ist, eine rezente Errungenschaft darstellt und dass sich auch patriarchale Kulturen von Zugewanderten unter Bedingungen der demokratischen Integration und Partizipation in Richtung Gleichberechtigung bewegen können und müssen.

Funding Open access funding provided by University of Vienna.

Open Access Dieser Artikel wird unter der Creative Commons Namensnennung 4.0 International Lizenz veröffentlicht, welche die Nutzung, Vervielfältigung, Bearbeitung, Verbreitung und Wiedergabe in jeglichem Medium und Format erlaubt, sofern Sie den/die ursprünglichen Autor(en) und die Quelle ord- 
nungsgemäß nennen, einen Link zur Creative Commons Lizenz beifügen und angeben, ob Änderungen vorgenommen wurden.

Die in diesem Artikel enthaltenen Bilder und sonstiges Drittmaterial unterliegen ebenfalls der genannten Creative Commons Lizenz, sofern sich aus der Abbildungslegende nichts anderes ergibt. Sofern das betreffende Material nicht unter der genannten Creative Commons Lizenz steht und die betreffende Handlung nicht nach gesetzlichen Vorschriften erlaubt ist, ist für die oben aufgeführten Weiterverwendungen des Materials die Einwilligung des jeweiligen Rechteinhabers einzuholen.

Weitere Details zur Lizenz entnehmen Sie bitte der Lizenzinformation auf http://creativecommons.org/ licenses/by/4.0/deed.de.

\section{Literatur}

AfD (Alternative für Deutschland) (2016): Programm für Deutschland. Das Grundsatzprogramm der Alternative für Deutschland. Stuttgart.

Bauer, Thomas (2018): Die Vereindeutigung der Welt. Über den Verlust an Mehrdeutigkeit und Vielfalt. Stuttgart.

Beck, Ulrich (1993): Die Erfindung des Politischen. Frankfurt am Main.

Büro HC Strache (2006): Widerliche sexistische Pseudopointen Jörg Haiders. OTS Originaltext Presseaussendung, https://www.ots.at/presseaussendung/OTS_20060624_OTS0003/fpd-widerlichesexistische-pseudopointen-joerg-haiders (zuletzt abgerufen am 3.1.2020).

Dietze, Gabriele (2018): Rechtspopulismus und Geschlecht. Paradox und Leitmotiv. In: Femina Politica. Jahrgang 27 (2018, 1), S. 34-46.

Duffek, Tatjana (2011): »Die Männer schaffen an« - Zwischen Discohäschen und Mutterkreuz: Was denken rechte Männer über Frauen. In: News, 5.11.2011, https://www.news.at/a/frauenbild-fpoe-bzoeein-mann-310996 (zuletzt abgerufen am 3.1.2020).

Eitz, Thorsten/Engelhardt, Isabelle (2015): Diskursgeschichte der Weimarer Republik. Band 1. Mit einem Vorwort von Georg Stötzel. Hildesheim/Zürich/New York.

Falter, Matthias/Stern, Verena (2019): Zum »Schutz des Volkes « gegen Gender. Eine geschlechterpolitische Verortung der FPÖ. In: Forschungsgruppe Ideologien und Politiken der Ungleichheit (Wien) (Hg.): Rechtsextremismus. Band 3: Geschlechterreflektierte Perspektiven. Wien, S. 185-204.

FPÖ (2013): Parteiprogramm der Freiheitlichen Partei Österreichs. In: Kotanko, Christoph (Hg.): Die Qual der Wahl. Die Programme der Parteien im Vergleich. Wien. S. 111-123.

FPÖ (2017): Österreicher verdienen Fairness. Freiheitliches Wahlprogramm zur Nationalratswahl 2017. Wien.

HC Strache, ehemalige Facebook-Seite http://facebook.com/strache (zuletzt abgerufen am 27.2.2019).

Howanietz, Michael (2013): Für ein freies Österreich. Herausgegeben von Norbert Hofer. Wien.

Jäger, Margret (1996): Fatale Effekte. Die Kritik am Patriarchat im Einwanderungsdiskurs. Duisburg.

Klein, Josef (1996): Insider-Lesarten. Einige Regeln zur latenten Fachkommunikation in Parteiprogrammen. In: Josef Klein/Hajo Diekmannshenke (Hg.): Sprachstrategien und Dialogblockaden. Linguistische und politikwissenschaftliche Studien zur politischen Kommunikation. Berlin/New York, S. 201-209.

Koppetsch, Cornelia (2019): Die Gesellschaft des Zorns. Rechtspopulismus im globalen Zeitalter. Bielefeld.

Madner, Martina (2018): Sind Sie Feminist? Ist Feminismus Frauensache? Nein - fünf Spitzenpolitiker machen das Thema mehr und auch weniger zum ihrigen. In: Wiener Zeitung Online, https://www. wienerzeitung.at/nachrichten/politik/oesterreich/951344-Sind-Sie-Feminist.html?em_no_split=1 (zuletzt abgerufen am 3.1.2020).

Mayer, Stefanie/Goetz, Judith (2019): Mit Gott und Natur gegen geschlechterpolitischen Wandel. In: Forschungsgruppe Ideologien und Politiken der Ungleichheit (Wien) (Hg.): Rechtsextremismus. Band 3: Geschlechterreflektierte Perspektiven. Wien, S. 205-247.

Mudde, Cas (2004): The Populist Zeitgeist. In: Government and Opposition 39 (4), S. 541-563.

Mudde, Cas (2007): Populist Radical Right Parties in Europe. Cambridge u. a.

Obermaier, Frederik/Obermayer, Bastian (2019): Die Ibiza-Affäre. Innenansichten eines Skandals. Köln.

Olschanski, Reinhard (2017): Der Wille zum Feind. Über populistische Rhetorik. Paderborn.

Pfaller, Robert (2008): Ästhetik der Interpassivität. Hamburg. 
Priester, Karin (2007): Populismus. Historische und aktuelle Erscheinungsformen. Frankfurt a.M./New York.

Priester, Karin (2012): Rechter und linker Populismus. Annäherung an ein Chamäleon. Frankfurt a.M./New York.

Reisigl, Martin (2002): »Dem Volk aufs Maul schauen, nach dem Mund reden und angst und bange machen« - Von populistischen Anrufungen, Anbiederungen und Agitationsweisen in der Sprache österreichischer PolitikerInnen. In: Eismann, Wolfgang (Hg.): Rechtspopulismus. Österreichische Krankheit oder europäische Normalität? Wien, S. 149-198.

Reisigl, Martin (2012a): Rechtspopulistische und faschistische Rhetorik - Ein Vergleich. In: Totalitarismus und Demokratie / Totalitarianism and Democracy. Zeitschrift für internationale Diktatur- und Freiheitsforschung / An International Journal for the Study of Dictatorship and Liberty. 9. Jahrgang / Heft 2 (2012): Populismus und Faschismus / Populism and Fascism. Göttingen, S. 303-323.

Reisigl, Martin (2012b): Zur kommunikativen Dimension des Rechtspopulismus. In: Sir Peter Ustinov Institut/Anton Pelinka/Birgitt Haller (Hg.): Populismus - Herausforderung oder Gefahr für die Demokratie? Wien, S. 141-162.

Reisigl, Martin (2014): Österreichischer Rechtspopulismus im Zeitalter von Mediendemokratie und medialer Erlebnisgesellschaft. In: Franz Januschek/Martin Reisigl (Hg.): Osnabrücker Beiträge zur Sprachtheorie (OBST) 86: Populismus in der digitalen Mediendemokratie. Duisburg, S. 71-99.

Reisigl, Martin (2016): Persuasive Tropen. Zur argumentativen Funktion semantischer Figuren. In: Germanistik in der Schweiz. Zeitschrift der Schweizerischen Akademischen Gesellschaft für Germanistik. Heft 13/2016. S. 37-53.

Rösslhumer, Maria (1999): Die FPÖ und die Frauen. Wien.

Rossmann, Eva (1995): Unter Männern. Frauen im österreichischen Parlament. Wien/Bozen.

Sauer, Birgit (2017): Gesellschaftstheoretische Überlegungen zum europäischen Rechtspopulismus. Zum Erklärungspotenzial der Kategorie Geschlecht. In: Politische Vierteljahresschrift, Jahrgang 58 (2017/1), S. 1-20.

Scharsach, Hans-Henning (2017): Stille Macht-Ergreifung. Hofer, Strache und die Burschenschaften. Wien.

SORA (2019): Wahlanalyse Nationalratswahl 2019. Wien. https://www.sora.at/fileadmin/downloads/ wahlen/2019_NRW_Wahlanalyse.pdf (zuletzt abgerufen am 3.1.2020).

SOS-Mitmensch (2018): Antimuslimischer Rassismus in der österreichischen Politik. Bericht 2018, https:// www2.sosmitmensch.at/dl/OMulJKJKNmKJqx4KJK/Bericht2018_Antimuslimischer_Rassismus_ in_der_Politik_SOS_MItmensch.pdf (zuletzt abgerufen am 3.1.2020).

Spier, Tim (2010): Modernisierungsverlierer? Die Wählerschaft rechtspopulistischer Parteien in Westeuropa. Wiesbaden.

Vorarlberg online (2013). Sexismus-Eklat. FP-Chef Strache erhitzt mit Posting die Gemüter, https://www. vol.at/sexismus-eklat-fp-chef-strache-erhitzt-mit-posting-die-gemueter/3475588 (zuletzt abgerufen am 3.1.2020). 